\title{
Screening and identification of NOTCH1, CDKN2A, and NOS3 as differentially expressed autophagy-related genes in erectile dysfunction
}

\author{
Chao Luo ${ }^{\text {Equal first author, } 1}{ }^{1}$, Xiongcai Zhou Equal first author, ${ }^{1,2}$, Li Wang $^{3}$, Qinyu Zeng ${ }^{1}$, Junhong Fan ${ }^{1}$, Shuhua He ${ }^{1}$, Haibo

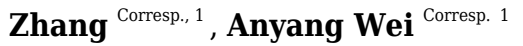 \\ 1 Department of urology, Nanfang hospital, Southern Medical University, Guangzhou, Guangdong province, China \\ 2 Department of urology, Guangzhou Eighth People's Hospital, Guangzhou Medical University, Guangzhou, Guangdong, China \\ ${ }^{3}$ School of basic medical science, Southern Medical University, Guangzhou, Guangdong, China \\ Corresponding Authors: Haibo Zhang, Anyang Wei \\ Email address: hai516@163.com, profwei@126.com
}

Background: Loss of function of key autophagy genes are associated with a variety of diseases. However, the specific role of autophagy-related genes in erectile dysfunction (ED) remains unclear. This study explores the autophagy-related differentially expressed genes (ARGs) profiles and related molecular mechanisms in Corpus Cavernosum endothelial dysfunction, which is a leading cause of ED. Methods: The Gene Expression Omnibus (GEO) database was used to identify the key genes and pathways. Differentially expressed genes (DEGs) were mined using the limma package in $R$ language. Next, ARGs were obtained by matching DEGs and autophagy-related genes from GeneCard using Venn diagrams. Gene Ontology (GO) and Kyoto Encyclopedia of Genes and Genomes (KEGG) analyses of ARGs were described using clusterProfiler and org.Hs.eg.db in R.Moreover, hub ARGs were screened out through protein-protein interaction (PPI), gene-microRNAs, and gene- transcription factors (TFs) networks then visualized using Cytoscape. Of note, the rat model of diabetic ED was established to validate some hub ARGs with qRT-PCR and Western blots. Results: Twenty ARGs were identified from four ED samples and eight nonED samples. GO analysis revealed that molecular functions (MF) of upregulated ARGs were mainly enriched in nuclear receptor activity. Also, MF of downregulated ARGs were mainly enriched in oxidoreductase activity, acting on $\mathrm{NAD}(\mathrm{P}) \mathrm{H}$ and heme proteins as acceptors. Moreover, six hub ARGs were identified by setting high degrees in the network. Additionally, hsa-mir-24-3p and hsa-mir-335-5p might play a central role in several ARGs regulation, and the transcription factors-hub genes network was centered with 13 ARGs. The experimental results further showed that the expression of Notch1, NOS3, and CDKN2A in the diabetic ED group was downregulated compared to the control.

Conclusions: Our study deepens the autophagy-related mechanistic understanding of 
endothelial dysfunction of ED. NOTCH1, CDKN2A, and NOS3 are involved in the regulation of endothelial dysfunction and may be potential therapeutic targets for ED by modulating autophagy. 
1 Screening and Identification of NOTCH1, CDKN2A,

2 and NOS3 as differentially expressed autophagy-

3 related genes in erectile dysfunction

4 Chao Luo ${ }^{1 *}$, Xiongcai Zhou ${ }^{12^{*}}$, Li Wang ${ }^{3 *}$, Qinyu Zeng ${ }^{1}$, Junhong Fan ${ }^{1}$, Shuhua He ${ }^{1}$, Haibo

5 Zhang ${ }^{1 \#}$, Anyang Wei ${ }^{1 \#}$

$6{ }^{1}$ Department of Urology, Nanfang Hospital, Southern Medical University, Guangzhou, 510515,

7 P.R. China.

8 2Department of urology, Guangzhou Eighth People's Hospital, Guangzhou Medical University,

9 Guangzhou, 510515, P.R. China.

$10{ }^{3}$ School of basic medical science, Southern Medical University, Guangzhou, 510515, P.R. China.

$11 *$ Equivalent contribution

12 \# Corresponding Authors

14 Corresponding Authors:

15 Anyang Wei, MD, PhD, Zhang-haibo, MD, PhD, Department of Urology, Nanfang Hospital,

16 Southern Medical University, 510515, Guangzhou, Guangdong, China. Tel: 020-61641765; Fax:

17 020-6161763; E-mail: profwei@126.com, hai516@163.com

\section{Abstract}

Background: Loss of function of key autophagy genes are associated with a variety of diseases.

However, the specific role of autophagy-related genes in erectile dysfunction (ED) remains unclear. This study explores the autophagy-related differentially expressed genes (ARGs) profiles and related molecular mechanisms in Corpus Cavernosum endothelial dysfunction, which is a leading cause of ED.

Methods: The Gene Expression Omnibus (GEO) database was used to identify the key genes and pathways. Differentially expressed genes (DEGs) were mined using the limma package in $\mathrm{R}$ language. Next, ARGs were obtained by matching DEGs and autophagy-related genes from 
Genomes (KEGG) analyses of ARGs were described using clusterProfiler and org.Hs.eg.db in R. Moreover, hub ARGs were screened out through protein-protein interaction (PPI), genemicroRNAs, and gene- transcription factors (TFs) networks then visualized using Cytoscape. Of note, the rat model of diabetic ED was established to validate some hub ARGs with qRT-PCR and Western blots.

Results: Twenty ARGs were identified from four ED samples and eight non-ED samples. GO analysis revealed that molecular functions (MF) of upregulated ARGs were mainly enriched in nuclear receptor activity. Also, MF of downregulated ARGs were mainly enriched in oxidoreductase activity, acting on $\mathrm{NAD}(\mathrm{P}) \mathrm{H}$ and heme proteins as acceptors. Moreover, six hub ARGs were identified by setting high degrees in the network. Additionally, hsa-mir-24-3p and hsa-mir-335-5p might play a central role in several ARGs regulation, and the transcription factors-hub genes network was centered with 13 ARGs. The experimental results further showed that the expression of Notch1, NOS3, and CDKN2A in the diabetic ED group was downregulated compared to the control.

Conclusions: Our study deepens the autophagy-related mechanistic understanding of endothelial dysfunction of ED. NOTCH1, CDKN2A, and NOS3 are involved in the regulation of endothelial dysfunction and may be potential therapeutic targets for ED by modulating autophagy.

\section{Introduction}

Erectile dysfunction (ED), also known as impotence, is the inability to maintain enough erections to achieve the satisfactory sexual activity (Muneer et al. 2014). ED is a common disease with high incidence worldwide, and there are few therapeutic methods. It is associated with aging, diabetes, and postoperative complications. The etiology of ED is mainly vascular, caused by nitric oxide (NO) metabolism disorder in the cells. NO enhancing drugs like phosphodiesterase 5 inhibitors (PDE-5i), which alleviate NO's decay, are widely utilized but still fail sometimes (Altabas \& Altabas 2015). The dysfunction of endothelial cells (ECs) plays a vital role in the pathophysiological process of ED. The complex pathogenesis of diabetic ED, including nerves, 
56

blood vessels, endothelium, smooth muscle, other tissues, and organs, have been long studied. As an independent factor, hyperglycemia leads to vascular endothelial cell injury and dysfunction. Vascular endothelial integrity and barrier function are destroyed through oxidative stress injury, leading to vascular disease.

Autophagy is an evolutionarily conserved eukaryotic self-catabolic mechanism. It balances energy sources, clears misaggregated or folded proteins, and clears damaged organelles (such as mitochondria, endoplasmic reticulum, and peroxisome). It is involved in many biological functions including development, in response to nutrient stress, cellular differentiation, and resistance to pathogens. Therefore, autophagy serves as a survival mechanism, although its deregulation is a related to non-apoptotic cell death. There is growing evidence that mutations or suppression of key autophagy genes are associated with cancer, neuropathy, cardiovascular disease, autoimmune diseases, and other diseases (Glick et al. 2010; Levine \& Kroemer 2019). Studies have confirmed that endothelial autophagy plays a critical role in the occurrence and development of cavernous endothelial cell lesions in ED (Lin et al. 2018; Zhang et al. 2019a). However, the role and specific regulatory mechanism of the autophagy gene in ECs remains unclear.

In this study we expected to analyze the human ED dataset in the GEO database using bioinformatics to explore the autophagy-related genes and related molecular mechanisms connected with endothelial dysfunction. Then, NOTCH1, PPARG, NOS3, KEAP1, CDKN2A, and IRS1 were predicted to affect endothelial dysfunction of ED as autophagy-related hub genes. Additionally, NOTCH1, CDKN2A, and NO3 were validated by establishing rat model of diabetic ED (DMED), and may be the underlying target molecules to treat ED by modulating autophagy.

Materials \& Methods

ED Datasets

Microarray data were obtained from the Gene Expression Omnibus (GEO) database 
83 (http://www.ncbi.nlm.nih.gov/geo) (Edgar et al. 2002). Specifically, we downloaded the gene expression profile of GSE10804 (Affymetrix GPL571platform, Affymetrix Human Genome U133A 2.0 Array) (Table 1). This dataset contained four samples of human corpus cavernosum ECs from donors with ED, one sample of human corpus cavernosum ECs from donors without ED, and seven samples of human arteriovenous ECs from donors without ED (three human umbilical vein ECs and four human coronary artery ECs).

RNA quality was measured using the "RNA degradation map" provided by the Affy package in R language (4.0.0) (Gautier et al. 2004; Wilson \& Miller 2005). The dataset was standardized usung the limma package in $\mathrm{R}$.

\section{Identification of differentially expressed genes (DEGs)}

The dataset was classified into two groups: the ED group (four samples of human corpus cavernosum ECs from donors with ED) and the non-ED group (including one sample of human corpus cavernosum ECs from donors without ED, and seven samples of human arteriovenous ECs from donors without ED). The limma package in $\mathrm{R}$ was used with the Wilcoxon test to screen out the significant DEGs. According to the annotation information of GPL571 platform, the probes were transformed to the corresponding gene symbol. Probe sets without corresponding gene symbols were excluded and genes with more than one probe set were averaged. The cut-off values were set according to the parameters, an absolute $\operatorname{logFC}>0.5$, and false discovery rate $(\mathrm{FDR})<0.05$.

\section{Identification of autophagy-related DEGs (ARGs)}

We obtained the autophagy-related genes (AREs) from GeneCard (https://www.genecards.org/). We entered the keywords "autophagy", used the relevance score $>5.0$ as the cutoff value, and downloaded them in the txt file format. Then, the DEGs and autophagy-related genes were entered into a Venn diagram (http://bioinformatics.psb.ugent.be/webtools/Venn/) to obtain the ARGs (Wang et al. 2014).

\section{Enrichment analysis and gene-concept network}

We explored the correlation between all ARGs, up-regulated ARGs, down-regulated ARGs, and 
110 AREs using R. We focused on the functional enrichment analysis of Gene Ontology (GO),

111 including the biological process, cellular component, and molecular function. We also analyzed

112 the Kyoto Encyclopedia of Genes and Genomes (KEGG) of 20 ARGs.

113 We introduced the "cnetplot" to depict the linkages of genes and biological concepts of GO to

114 form the Gene-Concept network (Yu et al. 2012).

115 Interaction network of hub genes

116 The ARGs interaction network was created in STRING (http://string-db.org/cgi/input.pl) using

117 the PPI network with the cutoff criteria as a combined score $>0.4$. Cytoscape (3.7.2) software

118 was used to analyze and visualize the PPI network for hub genes with a cutoff value of $>4$.

119 Construction of target gene-miRNA network and target gene- transcription factor (TF)

120 network

121 The expression of target genes in the post-transcriptional stage was regulated by miRNA or TF

122 under specific disease conditions. (Baldwin, 2001; Liu et al., 2020; Soifer et al., 2007)

123 NetworkAnalyst (https://www.networkanalyst.ca/) was used to analyze gene-TF interaction

124 (ENCODE database) and gene-miRNA interaction (miRTarBase v8.0 database) (Xia et al., 2015)

125 and was visualized using Cytoscape.

126 Animal and experiment designs

127 Eight-week old male Sprague-Dawley (SD) rats, weighing approximatley 200 grams, with

128 normal erectile function were provided by the Experimental Animal Center of Southern Medical

129 University. The Institutional Research Ethics Committee of Nanfang Hospital of Southern

130 Medical University provided full approval for this research (NFYY-2020-64). The 3R principle

131 was used in our animal experiments: reduction of animal numbers, replacement of animals using

132 other methods, and refinement of animal welfare. The experimental rats were randomly divided

133 into the DMED group and the control group (NC). The rats with DMED were modeled and

134 identified as previously mentioned (Zhang et al. 2019b). Briefly, healthy adult male SD rats were

135 intraperitoneally injected with a $1 \%$ streptozotocin solution $\left(65 \mathrm{mg} \mathrm{kg}^{-1}\right)$. Diabetes was

136 determined by measuring random tail vein blood glucose levels $72 \mathrm{~h}$ after injection. Rats with

PeerJ reviewing PDF | (2021:04:59773:1:0:NEW 8 Jul 2021) 
137

138

139

140

141

142

143

144

145

146

147

148

149

150

151

152

153

154

155

156

157

158

159

160

161

162

163

random blood glucose concentrations $>16.7 \mathrm{mmol} \mathrm{L}^{-1}$ were diagnosed as diabetic. Six DMED rats were included in this study; six control rats were not treated. We used a blood glucose meter (Anwen, Changsha, China) to measure random blood glucose in the tail vein blood every two weeks. Weight was also evaluated every two weeks.

All rats were reared in the Specific Pathogen Free (SPF) temperature-controlled animal house of Nanfang hospital's animal center with a 12-hour light-dark cycle. A specialized breeder changed the padding once a day and fed the rats with warm water that had been boiled and ordinary food (produced by Yancheng Biotechnology Co., Ltd., Guangzhou, China).

\section{Erectile function evaluation}

The mean ICP and ICP/MAP ratio were applied to evaluate erectile function, as mentioned above (Zhang et al. 2017). All rats were anesthetized with an intraperitoneal injection of sodium pentobarbital $(30 \mathrm{mg} / \mathrm{kg})$. The cavernous nerves were completely exposed and the corpus cavernosum was carefully isolated. A $25-\mathrm{G}$ needle containing a $100 \mathrm{U} / \mathrm{ml}$ heparin solution was slowly inserted into proximal corpus cavernosum to ensure that the rat was securely connected with the sensor and amplifier (MP150 Biopac System, Biopac Systems Inc., CA, USA). Both were connected using AcqKnowledge ${ }^{\circledR}$ (V4.4) software. We recorded the intracavernous pressure (ICP) when the cavernous nerve was stimulated with bipolar stainless steel electrodes. The mean atrial blood pressure (MAP) was measured by intubation in the biological signal system above after exposing the left carotid artery. A successful DMED rat model should meet an ICP of less than $60 \mathrm{mmHg}$ and a ICP/MAP ratio less than 0.5 . Data were recorded and the penis shaft was collected for future study. All rats were euthanized using a 10-fold anesthetic dose $(300 \mathrm{mg} / \mathrm{kg}$ sodium pentobarbital).

\section{Histology}

Freshly dissected tissue was fixated. H\&E and Masson's trichrome staining was performed to determine tissue structure changes based on the manufacturer's instructions. We also determined the ratio between smooth muscle and collagen in the corpus cavernosum. Images were captured using Olympus BX63 microscopy (Olympus, Tokyo, Japan). 
164

165

166

167

168

169

170

171

172

173

174

175

176

177

178

179

180

181

182

183

184

185

186

187

188

189

190

\section{Immunofluorescence analysis and laser confocal microscope}

Tissue slides were prepared in a smiliar manner to those used in H\&E staining. After antigen repair, goat serum was inoculated at room temperature for $30 \mathrm{~min}$, the CD31 antibody (Abcam, ab182981) was incubated overnight, and a secondary antibody solution of Goat Anti-Rabbit IgG (HRP) (Abcam, ab205718) was added. Antigen repair was performed after the first dyeing and cleaning. Donkey serum was inoculated at room temperature for $30 \mathrm{~min}$, the LC3 antibody (1:200, Proteintech, USA) was incubated overnight, and Donkey Anti-Rabbit 594 secondary antibody (Abcam, ab150075) was added through a drip. We used 4', 6-Diamidino-2phenylindole (DAPI, Solarbio, \#C0060) to stain the cell nuclei and images were captured using laser confocal microscopy (Nikon, Tokyo, Japan).

\section{Real-time quantitative PCR (qRT-PCR)}

Cavernous tissue RNA was extracted and used for qRT-PCR analysis. The primer sequences we used were: $\beta$-Actin, sense: 5'-GATCA-AGATCATTGCTCCTCCTG-3', anti-sense: 5'-

AGGGTGTAAAACGCAG-CTCA-3'; NOTCH1, sense: 5'-CAGTACAACCCGCTAAGGC, anti-sense: 5'-GGACAAGG-TATTGGTGGAGA-3'; NOS3, sense: 5'-

CCGATTACACGACATTG-AGA-3', anti-sense: 5'-TGGTCCAGTTGGG-AGCAT-3' (antisense); KEAP1, sense: 5'-GGTCGC-CCTGTGCCTCTAT-3', anti-sense: 5'-

CACGCTGCTGTGGTGGAT-3'; CDKN2A, sense: 5'-GAGGGCTTCCTAGACACTCTG-3', anti-sense: 5'-CGCAAATACCGCA-CGAC-3'; PPARG, sense: 5'-

CCTCCCTGATGAATAAAGA-3', anti-sense: 5'-AAC-TCAAACTTAGGCTCCA-3'; IRS1, sense: 5'-GAGTGGTGGAGTTGAGTTGG-3', anti-sense: 5'-GTCCGCATGTCAGCATA-3'.

\section{Western blot (WB)}

WB was carried out for protein expression analysis as previously described (He et al., 2012). We detected LC3 and Beclin-1 using a rabbit anti-LC3 antibody (Proteintech, 14600-1-AP) (1:1,000), anti-Beclin-1 antibody (Proteintech, 11306-1-AP) (1:1,000), anti-NOTCH1 antibody (Proteintech, 10062-2-AP) (1:1000), and anti-eNOS (Abcam, ab199956) (1:1000). Secondary antibodies were purchased from LI-COR Biosciences (catalog \#D00825-14, USA) and were 
191

192

193

194

195

196

197

198

199

200

201

202

203

204

205

206

207

208

209

210

211

212

213

214

215

216

217

diluted at 1:15,000. $\beta$-actin (ABclonal, AC026) was used as a loading control.

\section{Statistical analysis}

The gray value and fluorescence intensity of image were further analyzed using Image $\mathbf{J}$ software. The outcomes were shown as the mean \pm standard error. One-way ANOVA was applied to perform statistical comparisons among the groups. R software (version 4.0.0) and GraphPad Prism 8.3.0 were used to perform all statistical analyses. A P-value of $<0.05$ was considered statistically significant.

\section{Results}

\section{Identification of differentially expressed autophagy-related genes (ARGs)}

We retrieved a total of four ED samples and eight non-ED samples with gene expression profiles from the GEO dataset. The RNA degradation plot was introduced to measure the quality of the RNA in the raw-data sample. The fluorescence intensity at the 5 ' end of the chip was much lower than that at the $3^{\prime}$ end, and the slopes of the curves ranked in a similar order (Fig. 1A). We normalized the raw data for further analysis (Fig. 1B). We identified 1,236 significant DEGs between the ED and non-ED samples. Among these DEGs, 423 genes were up-regulated in ED tissue compared with non-ED tissue; the other 815 genes were down-regulated via Volcano Plot and heatmap (Fig. 1C). We matched DEGs with 380 AREs from the GeneCard (Supplemental File 1) using a Venn diagram (Fig. 1D), resulting in 20 ARGs. Thirteen ARGs were downregulated in ED tissues; the other seven genes were up-regulated compared with non-ED tissues (Table 2, Fig. 1E).

\section{Functional enrichment analysis and Interactions of ARGs}

All twenty ARGs were used for functional enrichment analysis to explore the biological functions and pathways of ARGs in autophagy. GO results showed that the biological process (BP) of ARGs were mainly enriched in autophagy, a process utilizing autophagic mechanism, the regulation of autophagy, positive regulation of proteolysis, and the positive regulation of the cellular catabolic process. The cellular component of ARGs was enriched in the nuclear outer membrane. ARGs' molecular functions were enriched with oxidoreductase activity, acting on 
$218 \mathrm{NADH}$, heme protein as acceptor, enzyme inhibitor activity, Hsp70 protein binding, ligand-

219 activated transcription factor activity, and nuclear receptor activity (Figs. 2A, 2C). The results of

220 KEGG enrichment of ARGs were significantly enriched in the mTOR signaling pathway, PI3K-

221 Akt signaling pathway, longevity regulating pathway, AMPK signaling pathway, and platelet

222 activation, excluding autophagy-related pathways (Fig. 2B).

223 The enriched GO pathways of molecular functions of seven up-regulated ARGs were primarily

224 nuclear receptor activity, ligand-activated transcription factor activity, and steroid hormone

225 receptor activity (Fig. 2D). GO enrichment of 13 down-regulated ARGs showed that molecular

226 functions were enriched in oxidoreductase activity, acting on $\mathrm{NAD}(\mathrm{P}) \mathrm{H}$, heme protein as

227 acceptor, enzyme inhibitor activity, and Hsp70 protein binding (Fig. 2E). The GO enrichment of

228380 AREs showed that molecular functions were enriched in ubiquitin-protein ligase binding,

229 ubiquitin-like protein ligase binding, protein serine/threonine kinase activity, phosphatase

230 binding, protein phosphatase binding, and heat shock protein binding (Fig. 2F). Interestingly,

231 heat shock protein (HSP) binding appeared in three groups.

232 Construction of the PPI network, gene-microRNA network, and gene-TF network

233 We explored the twenty ARGs interactions using the STRING website (Fig. 3A). The gene

234 network demonstrated that IRS1, CDKN2A, NOTCH1, PPARG, KEAP1, and NOS3 were the

235 hub genes using Cytospace software (Fig. 3B). The top three ARGs for miRNAs were AR,

236 NGFR, and FLCN. Hsa-mir-24-3p and Hsa-mir-335-5p may control the largest number of ARGs

237 (Fig. 3C). NOS3, VAMP8, and MLST8 were the top three target ARGs for TFs, modulated by

238 78, 77, and 70 TFs (Fig. 3D).

239 Blood glucose, weight, ICP/MAP, and morphological changes of penis corpus cavernosum

240 of DMED model

241 The blood glucose of DMED rats was significantly higher than that of the NC rats, while the

242 weight of DMED rats was significantly lower than that of the NC rats at the 8th week after

243 modeling (Fig.4A). We successfully established the DMED rat model with ICP less than 60

$244 \mathrm{mmHg}$ and ICP/MAP ratio less than 0.5 (Fig. 4B). Results from H\&E and Masson's trichrome

PeerJ reviewing PDF | (2021:04:59773:1:0:NEW 8 Jul 2021) 
245

246

247

248

249

250

251

252

253

254

255

256

257

258

259

260

261

262

263

264

265

266

267

268

269

270

271

staining revealed that the corpus cavernosum was disordered and the ratio of fibroblast to collagen decreased when compared with the NC group. The endothelium and smooth muscle layer in the DMED group were thinner than the NC group (Figs. 4C-4D).

\section{Enhanced autophagy of cavernous tissue and Endothelium in DMED model}

The protein level of LC3-II and LC3-I, and the ratio of LC3-II/LC3-I were upregulated, and the grayscale value of Beclin-1 was downregulated in DMED group compared with the NC group (Fig. 5A). The fluorescence intensity of LC3 in the DMED group was significantly higher than that in the NC group. The increase of LC3 represented an increase in autophagosomes. The discontinuity of CD31 labeled ECs, the decrease of fluorescence intensity, and the disappearance of fluorescence on smooth muscle surface suggested that the ECs in DMED tissue were injured (Fig. 5B).

\section{The expression level of hub ARGs}

The results of qRT-PCR showed that the expressions of Notch1, PPARG, NOS3, CDKN2A, and IRS1 were down-regulated, however, there was no statistical significance in the expression of KEAP1 compared to NC group (Figs. 6A-F). The gene expression of NOTCH1, CDKN2A, and NOS3 was consistent with the data in Table 2. The protein level of NOTCH1, CDKN2A, and NOS3 were downregulated in DMED group compared to NC (Fig. 6G).

\section{Discussion}

Endothelial dysfunction is one of the leading causes of ED. ECs play a critical role in regulating inflammation, platelet aggregation, vascular smooth muscle hyperplasia, and thrombosis. Thus, targeting endothelial dysfunction may be a more effective treatment for ED. Studies have suggested that endothelial dysfunction may be alleviated by inhibiting autophagy (Altabas \& Altabas, 2015; Verma \& Anderson, 2002). Autophagy is a multi-step collaborative process regulated by autophagy-related genes (Sarkar et al., 2014). However, the pathway of autophagyrelated genes and ED remains unclear. In this study, we utilized bioinformatics to mine twenty autophagy-related genes and their related molecular functions in an ED database. Moreover, six hub genes were further screened out using the PPI co-expression network. We further verified 
272 these genes by constructing rat model of diabetic ED relying on the homology of human and 273 mammalian genes.

274 The LC3-II/LC3-I ratio and LC3-II/ $\beta$-actin ratio in the DMED group was relatively higher than 275 the control group. LC3-II is bound to the cell membrane in mature autophagosomes, is released 276 upon fusion with the lysosome, and is considered to be a marker for autophagy detection (Ni et 277 al., 2011). This suggests that autophagy is enhanced in DMED tissue. In addition, the enhanced 278 autophagy of endothelial cells was confirmed by CD31 and LC3 co-staining. Excessive ROS accumulation promotes compensatory autophagy during the pathogenesis of diabetic ED, which protects the body from oxidative stress by clearing damaged intracellular substances (ScherzShouval et al., 2007). The Beclin1/ $\beta$-actin ratio in the DMED group was lower than in the control. This protein plays a central role in the formation and maturation of autophagosomes. The conditions that disrupt or promote the Beclin1-Bcl2 complex play a crucial part in determining whether cells undergo autophagy or apoptosis. A recent study showed that when Beclin1 was induced it facilitated autophagy in various cell types, including ECs (Leonard et al., 2019). We found that the gene expression of NOTCH1, CDKN2A, and NOS3 was consistent with the high throughput sequencing results from GEO. These may become underlying target molecules in the regulation of EC autophagy in ED.

Notch is a classical signaling pathway, including four Notch receptors (Notch 1, 2, 3 and 4) as well as five notch ligands (delta-like receptors 1, 3, 4 and jagged 1 and 2) in mammals (Gordon et al., 2008). The pathway is activated when a Notch receptor interacts with a ligand. Studies suggested that the inhibition of autophagy leads to the activation of Notch pathway. Autophagy suppresses the Notch1 pathway by up-regulating the degradation of Notch1 (Li et al., 2016; Wu et al., 2016; Zeng et al., 2016). The reduction of NOTCH1 in ECs is a predisposing factor for vascular inflammation and atherosclerosis (Briot et al., 2015). Endothelial dysfunction may be associated with a decrease of Notch1. Moreover, the Notch pathway was associated with senescence which the mainstream factor of ED. A recent study showed that the Notch-1 inhibitor, Tangeretin, alleviated ED by increasing the maximum ICP/MAP and up-regulating the 
299

300

301

302

303

304

305

306

307

308

309

310

311

312

313

314

315

316

317

318

319

320

321

322

323

324

325

expression of eNOS in hypertensive rats (Chiangsaen et al., 2020). Thus, NOTCH1 may be a candidate biomarker for ED.

CDKN2A, also called $\mathrm{p} 16^{\mathrm{INK} 4 \mathrm{a}}(\mathrm{p} 16)$, has been shown to increase with age in several rodent and human tissues (Baker et al., 2011). As a member of the INK4 family of cyclin-dependent kinase inhibitors, p16 plays a critical role in cell-cycle regulation. p16 expression inhibits cellular proliferation by downregulating cyclin-dependent kinases 4 and 6 (CDK4/6) (Serrano, 1997). As a tumor suppressor, $\mathrm{p} 16^{\mathrm{INK} 4 \mathrm{a}}(\mathrm{p} 16)$ is a well-studied maturation marker, inducing cellular senescence (permanent cell-cycle arrest) in response to stress. p16 accumulates in tissues with age, causing cellular senescence. The elimination of p16 expression in senescent cells is associated with a prolonged life span and a reduction of tumorigenesis (Baker et al., 2011). Studies have suggested that autophagy inhibitors bafilomycin A1 and chloroquine induced p16 accumulation in stagnant vesicles containing the autophagy marker LC3. The accumulation of p16 in these vesicles was consistent with the increase of LC3-II. The knockout of autophagosome chaperone p62 attenuated p16 aggregates in lysosomes, indicating that p16 targets these vesicles through p62. As the regulator of autophagy, p16 performs a key role in the etiology of cancer and dementia (Coryell et al., 2020). Moreover, advanced age is a critical risk factor for most chronic diseases and functional defects in humans. A significant increase has been shown in ED incidence in men over the age of 40 (Shamloul \& Ghanem, 2013). Senescence is closely associated with ED. We found that the expression of CDKN2A was decreased. This result suggests that not all cells with high expression of $\mathrm{p} 16^{\mathrm{Ink} 4 \mathrm{a}}$ are senescent cells and not every senescent cell has high expression of p16 $6^{\mathrm{Ink} 4}$ (Hall et al., 2017). There should be further studies conducted on CDKN2A as an autophagy-related molecule.

Endothelial nitric oxide synthase (eNOS) is also known as NOS3, one of three subtypes of nitric oxide synthase (NOS). It is involved in the synthesis of nitric oxide (NO) with L-arginine and molecular oxygen as substrates. It is also involved in the regulation of physiological and pathological functions. The pathophysiology of endothelial dysfunction involves multiple mechanisms, such as the dysregulation of NO by vascular/endothelial eNOS in ED. 
326

327

328

329

330

331

332

333

334

335

336

337

338

339

340

341

342

343

344

345

346

347

348

349

350

351

352

eNOS performs an essential role in erectile response. eNOS activity and endothelial NO bioavailability in the penis are jointly regulated by multiple post-translational molecular mechanisms, including eNOS phosphorylation, eNOS interaction with upstream proteins and contractile pathways, and the generation of reactive oxygen species (ROS). The activation of the $\mathrm{PI} 3 \mathrm{~K} / \mathrm{Akt} / \mathrm{eNOS}$ pathway was considered to be one of classic pathways to regulate endothelial dysfunction and participate in the cellular and molecular biology of ECs (Chen et al., 2021; Chen et al., 2019; Duan et al., 2019; Zhang \& Zhang, 2020). The regulation of the AMPK pathway and the mTOR pathway corresponded to endothelial autophagy (Xiong et al., 2014). Our results confirmed that autophagosome increased in the cavernous ECs of DMED rats, suggesting an increase in autophagy. The participation of eNOS in the autophagy pathway in endothelial dysfunction requires further experimental study.

Previous literature reporting on mammalian cells has indicated that protein degradation pathways are commonly classified in the ubiquitin-proteasome system. There are three main types of autophagy: macro-autophagy, micro-autophagy, and chaperone-mediated autophagy. Hsp70 acts a central hub in protein degradation through the ubiquitin-proteasome system and different autophagy pathways (Fernández-Fernández et al., 2017). Our GO analysis suggested downregulated ARGs and 380 AREs were both associated with HSP protein binding. The Hsp70 family performs central roles in every aspect of proteostasis, from protein folding to disaggregation and degradation (Mayer \& Bukau, 2005). Targeting HSP70 binding in ED may be a new direction for future research. Furthermore, the upregulated ARGs (AR and PPARG) were mainly enriched in nuclear receptor activity and downregulated ARGs (TSC1 and SNCA) were mainly enriched in oxidoreductase activity. However, their relationship with ED requires further study.

The top three targeted ARGs in the miRNA-gene network were AR, NGFR, and FLCN. It has been suggested that nerve growth factor (NGF) may induce nerve regeneration by activating the autophagy of Schwann cells. It may also promote the maintenance, survival, and function of vascular endothelial cells (Tanaka et al., 2004). Low doses of rapamycin, an autophagic inducer, 
353

354

355

356

357

358

359

360

361

362

363

364

365

366

367

368

369

370

371

372

373

374

375

376

377

378

379

accelerated autophagy by activating the NGFR promoter. Therefore, NGFR may be involved in the regulation of endothelial autophagy. Hsa-mir-24-3p and Hsa-mir-335-5p, which control more ARGs, perform a key role in angiogenesis (Esquinas et al., 2017; Li et al., 2020). The relationship between AR, FLCN, and endothelial dysfunction requires further study. NOS3, VAMP8, and MLST8 were ranked as the top three targeted ARGs in the TF-gene network. A previous study showed that platelets stimulated BMDC homing and promoted angiogenesis. Thus they are a key mediator between hypoxic tissue and bone marrow, in which VAMP8 plays an important role (Feng et al., 2011). In endothelial cells, MLST8 is involved in modulating the activation of Akt and reducing the phosphorylation of Akt targets such as the eNOS and FOXO subfamily (Partovian et al., 2008). The role of NOS3 in endothelial dysfunction has been discussed.

Recently there has been great interest in the relationship between autophagy and ED. Glucagonlike peptide-1 (GLP-1) receptor agonists, defocused low-energy shock wave therapy, and stem cell therapy have been suggested to alleviate ED by promoting endothelium autophay in DMED rats (Yuan et al., 2020; Zhang et al., 2019a; Zhu et al., 2018). Autophagy was shown in the ED model of BCNI and aging. Thus ED may be allieviated by improving autophagy (Tang et al., 2018; Ye et al., 2021). However, there is no research on the expression changes and molecular functions of ED-related autophagy genes. Our study may provide new directions for future research and novel molecular targets for the treatment of ED.

Our research was limited by the small number of microarray samples which may lead to statistical bias. Secondly, we screened genes based on microarray data sourced from human tissue in GEO. However, this was verified on SD rats which may cause species bias. We have also not specifically studied whether these genes can regulate autophagy.

\section{Conclusions}

Our study mined endothelial-related ARGs to identify the possible pathogenesis of ED related to autophagy. Consistent with the fact that high glucose accelerates the senescence of endothelial cells by inhibiting autophagy, two of three identified genes, NOTCH1 and CDKN2A, are 
380

381

382

383

384

385

386

387

388

389

390

391

392

393

394

395

396

397

398

399

400

401

402

403

404

405

406

407

408

409

410

411

412

413

414

415

416

417

involved in senescence. NOTCH1, CDKN2A, and NO3 may be the underlying therapeutic targets to treat ED. Moreover, the study of upstream transcription factors and miRNAs of target genes may improve the mechanistic understanding of endothelial dysfunction in ED.

\section{References}

Altabas V, and Altabas K. 2015. DPP-4 inhibition improves a sexual condition? Med Hypotheses 85:124126. 10.1007/s10517-017-3940-6

10.1016/j.mehy.2015.04.011

Baker DJ, Wijshake T, Tchkonia T, LeBrasseur NK, Childs BG, van de Sluis B, Kirkland JL, and van Deursen JM. 2011. Clearance of p16Ink4a-positive senescent cells delays ageing-associated disorders. Nature 479:232-236. 10.1038/nature10600

Baldwin AS, Jr. 2001. Series introduction: the transcription factor NF-kappaB and human disease. J Clin Invest 107:3-6. 10.1172/jci11891

Briot A, Civelek M, Seki A, Hoi K, Mack JJ, Lee SD, Kim J, Hong C, Yu J, Fishbein GA, Vakili L, Fogelman AM, Fishbein MC, Lusis AJ, Tontonoz P, Navab M, Berliner JA, and Iruela-Arispe ML. 2015. Endothelial NOTCH1 is suppressed by circulating lipids and antagonizes inflammation during atherosclerosis. $J$ Exp Med 212:2147-2163. 10.1084/jem.20150603

Chen J, Huang Y, Hu X, Bian X, and Nian S. 2021. Gastrodin prevents homocysteine-induced human umbilical vein endothelial cells injury via PI3K/Akt/eNOS and Nrf2/ARE pathway. J Cell Mol Med 25:345-357. 10.1111/jcmm.16073

Chen L, Qin L, Liu X, and Meng X. 2019. CTRP3 Alleviates Ox-LDL-Induced Inflammatory Response and Endothelial Dysfunction in Mouse Aortic Endothelial Cells by Activating the PI3K/Akt/eNOS Pathway. Inflammation 42:1350-1359. 10.1007/s10753-019-00996-1

Chiangsaen P, Maneesai P, Kukongviriyapan U, Tong-Un T, Ishida W, Prachaney P, and Pakdeechote P. 2020. Tangeretin ameliorates erectile and testicular dysfunction in a rat model of hypertension. Reprod Toxicol 96:1-10. 10.1016/j.reprotox.2020.05.012

Coryell PR, Goraya SK, Griffin KA, Redick MA, Sisk SR, and Purvis JE. 2020. Autophagy regulates the localization and degradation of p16(INK4a). Aging Cell 19:e13171. 10.1111/acel.13171

Duan MX, Zhou H, Wu QQ, Liu C, Xiao Y, Deng W, and Tang QZ. 2019. Andrographolide Protects against HG-Induced Inflammation, Apoptosis, Migration, and Impairment of Angiogenesis via PI3K/AKT-eNOS Signalling in HUVECs. Mediators Inflamm 2019:6168340. $10.1155 / 2019 / 6168340$

Edgar R, Domrachev M, and Lash AE. 2002. Gene Expression Omnibus: NCBI gene expression and hybridization array data repository. Nucleic Acids Res 30:207-210. 10.1093/nar/30.1.207

Esquinas C, Janciauskiene S, Gonzalo R, Mas de Xaxars G, Olejnicka B, Belmonte I, Barrecheguren M, Rodriguez E, Nuñez A, Rodriguez-Frias F, and Miravitlles M. 2017. Gene and miRNA expression profiles in PBMCs from patients with severe and mild emphysema and PiZZ alpha1antitrypsin deficiency. Int J Chron Obstruct Pulmon Dis 12:3381-3390. 10.2147/copd.S145445 
418 Feng W, Madajka M, Kerr BA, Mahabeleshwar GH, Whiteheart SW, and Byzova TV. 2011. A novel role

419

420

421

422

423

424

425

426

427

428

429

430

431

432

433

434

435

436

437

438

439

440

441

442

443

444

445

446

447

448

449

450

451

452

453

454

455

456

457

458 for platelet secretion in angiogenesis: mediating bone marrow-derived cell mobilization and homing. Blood 117:3893-3902. 10.1182/blood-2010-08-304808

Fernández-Fernández MR, Gragera M, Ochoa-Ibarrola L, Quintana-Gallardo L, and Valpuesta JM. 2017. Hsp70 - a master regulator in protein degradation. FEBS Lett 591:2648-2660. 10.1002/18733468.12751

Gautier L, Cope L, Bolstad BM, and Irizarry RA. 2004. affy--analysis of Affymetrix GeneChip data at the probe level. Bioinformatics 20:307-315. 10.1093/bioinformatics/btg405

Glick D, Barth S, and Macleod KF. 2010. Autophagy: cellular and molecular mechanisms. J Pathol 221:3-12. 10.1002/path.2697

Gordon WR, Arnett KL, and Blacklow SC. 2008. The molecular logic of Notch signaling--a structural and biochemical perspective. J Cell Sci 121:3109-3119. 10.1242/jcs.035683

Hall BM, Balan V, Gleiberman AS, Strom E, Krasnov P, Virtuoso LP, Rydkina E, Vujcic S, Balan K, Gitlin, II, Leonova KI, Consiglio CR, Gollnick SO, Chernova OB, and Gudkov AV. 2017. p16(Ink4a) and senescence-associated $\beta$-galactosidase can be induced in macrophages as part of a reversible response to physiological stimuli. Aging (Albany NY) 9:1867-1884. 10.18632/aging.101268

He SH, Wei AY, Yang Y, Hu YW, Luo XG, Liu Y, and Zhang T. 2012. Reduced expression of myocardin and serum response factor in the cavernous tissue of diabetic rats. Andrologia 44 Suppl 1:518-522. 10.1111/j.1439-0272.2011.01218.x

Leonard A, Millar MW, Slavin SA, Bijli KM, Dionisio Santos DA, Dean DA, Fazal F, and Rahman A. 2019. Critical role of autophagy regulator Beclin1 in endothelial cell inflammation and barrier disruption. Cell Signal 61:120-129. 10.1016/j.cellsig.2019.04.013

Levine B, and Kroemer G. 2019. Biological Functions of Autophagy Genes: A Disease Perspective. Cell 176:11-42. 10.1016/j.cell.2018.09.048

Li B, Duan P, Li C, Jing Y, Han X, Yan W, and Xing Y. 2016. Role of autophagy on bone marrow mesenchymal stem-cell proliferation and differentiation into neurons. Mol Med Rep 13:14131419. 10.3892/mmr.2015.4673

Li HM, Mo ZW, Peng YM, Li Y, Dai WP, Yuan HY, Chang FJ, Wang TT, Wang M, Hu KH, Li XD, Ning DS, Chen YT, Song YK, Lu XL, Pei Z, Dong YG, Wang ZP, Zhang X, Xu YQ, Wang SM, $\mathrm{Ou} \mathrm{ZJ}$, and Ou JS. 2020. Angiogenic and Antiangiogenic mechanisms of high density lipoprotein from healthy subjects and coronary artery diseases patients. Redox Biol 36:101642. 10.1016/j.redox.2020.101642

Lin H, Wang T, Ruan Y, Liu K, Li H, Wang S, Li M, and Liu J. 2018. Rapamycin Supplementation May Ameliorate Erectile Function in Rats With Streptozotocin-Induced Type 1 Diabetes by Inducing Autophagy and Inhibiting Apoptosis, Endothelial Dysfunction, and Corporal Fibrosis. J Sex Med 15:1246-1259. 10.1016/j.jsxm.2018.07.013

Liu Y, Jin J, Chen Y, Chen C, Chen Z, and Xu L. 2020. Integrative analyses of biomarkers and pathways for adipose tissue after bariatric surgery. Adipocyte 9:384-400. 10.1080/21623945.2020.1795434 Mayer MP, and Bukau B. 2005. Hsp70 chaperones: cellular functions and molecular mechanism. Cell Mol Life Sci 62:670-684. 10.1007/s00018-004-4464-6 
459 Muneer A, Kalsi J, Nazareth I, and Arya M. 2014. Erectile dysfunction. Bmj 348:g129. 10.1136/bmj.g129

460

461

462

463

464

465

466

467

468

469

470

471

472

473

474

475

476

477

478

479

480

481

482

483

484

485

486

487

488

489

490

491

492

493

494

495

496

497

498

499

Ni HM, Bockus A, Wozniak AL, Jones K, Weinman S, Yin XM, and Ding WX. 2011. Dissecting the dynamic turnover of GFP-LC3 in the autolysosome. Autophagy 7:188-204. 10.4161/auto.7.2.14181

Partovian C, Ju R, Zhuang ZW, Martin KA, and Simons M. 2008. Syndecan-4 regulates subcellular localization of mTOR Complex2 and Akt activation in a PKCalpha-dependent manner in endothelial cells. Mol Cell 32:140-149. 10.1016/j.molcel.2008.09.010

Sarkar C, Zhao Z, Aungst S, Sabirzhanov B, Faden AI, and Lipinski MM. 2014. Impaired autophagy flux is associated with neuronal cell death after traumatic brain injury. Autophagy 10:2208-2222. 10.4161/15548627.2014.981787

Scherz-Shouval R, Shvets E, Fass E, Shorer H, Gil L, and Elazar Z. 2007. Reactive oxygen species are essential for autophagy and specifically regulate the activity of Atg4. Embo j 26:1749-1760. 10.1038/sj.emboj.7601623

Serrano M. 1997. The tumor suppressor protein p16INK4a. Exp Cell Res 237:7-13. 10.1006/excr.1997.3824

Shamloul R, and Ghanem H. 2013. Erectile dysfunction. Lancet 381:153-165. 10.1016/s01406736(12)60520-0

Soifer HS, Rossi JJ, and Saetrom P. 2007. MicroRNAs in disease and potential therapeutic applications. Mol Ther 15:2070-2079. 10.1038/sj.mt.6300311

Tanaka A, Wakita U, Kambe N, Iwasaki T, and Matsuda H. 2004. An autocrine function of nerve growth factor for cell cycle regulation of vascular endothelial cells. Biochem Biophys Res Commun 313:1009-1014. 10.1016/j.bbrc.2003.12.036

Tang Z, Cui K, Luan Y, Ruan Y, Wang T, Yang J, Wang S, Liu J, and Wang D. 2018. Human tissue kallikrein 1 ameliorates erectile function via modulation of macroautophagy in aged transgenic rats. Andrology 6:766-774. 10.1111/andr.12512

Verma S, and Anderson TJ. 2002. Fundamentals of endothelial function for the clinical cardiologist. Circulation 105:546-549. 10.1161/hc0502.104540

Wang Y, Thilmony R, and Gu YQ. 2014. NetVenn: an integrated network analysis web platform for gene lists. Nucleic Acids Res 42:W161-166. 10.1093/nar/gku331

Wilson CL, and Miller CJ. 2005. Simpleaffy: a BioConductor package for Affymetrix Quality Control and data analysis. Bioinformatics 21:3683-3685. 10.1093/bioinformatics/bti605

Wu X, Fleming A, Ricketts T, Pavel M, Virgin H, Menzies FM, and Rubinsztein DC. 2016. Autophagy regulates Notch degradation and modulates stem cell development and neurogenesis. Nat Commun 7:10533. 10.1038/ncomms10533

Xia J, Gill EE, and Hancock RE. 2015. NetworkAnalyst for statistical, visual and network-based metaanalysis of gene expression data. Nat Protoc 10:823-844. 10.1038/nprot.2015.052

Xiong Y, Yepuri G, Forbiteh M, Yu Y, Montani JP, Yang Z, and Ming XF. 2014. ARG2 impairs endothelial autophagy through regulation of MTOR and PRKAA/AMPK signaling in advanced atherosclerosis. Autophagy 10:2223-2238. 10.4161/15548627.2014.981789

Ye M, Zhao F, Ma K, Zhou K, Ma J, Fu H, Xu Z, Huang W, Wang W, Zhao J, and Lv B. 2021. Enhanced effects of salidroside on erectile function and corpora cavernosa autophagy in a cavernous nerve

PeerJ reviewing PDF | (2021:04:59773:1:0:NEW 8 Jul 2021) 
injury rat model. Andrologia:e14044. 10.1111/and.14044

Yu G, Wang LG, Han Y, and He QY. 2012. clusterProfiler: an R package for comparing biological themes among gene clusters. Omics 16:284-287. 10.1089/omi.2011.0118

Yuan P, Ma D, Gao X, Wang J, Li R, Liu Z, Wang T, Wang S, Liu J, and Liu X. 2020. Liraglutide Ameliorates Erectile Dysfunction via Regulating Oxidative Stress, the RhoA/ROCK Pathway and Autophagy in Diabetes Mellitus. Front Pharmacol 11:1257. 10.3389/fphar.2020.01257

Zeng J, Jing Y, Shi R, Pan X, Lai F, Liu W, Li R, Gao L, Hou X, Wu M, and Wei L. 2016. Autophagy regulates biliary differentiation of hepatic progenitor cells through Notch1 signaling pathway. Cell Cycle 15:1602-1610. 10.1080/15384101.2016.1181234

Zhang C, Luo D, Li T, Yang Q, Xie Y, Chen H, Lv L, Yao J, Deng C, Liang X, Wu R, Sun X, Zhang Y, Deng C, and Liu G. 2019a. Transplantation of Human Urine-Derived Stem Cells Ameliorates Erectile Function and Cavernosal Endothelial Function by Promoting Autophagy of Corpus Cavernosal Endothelial Cells in Diabetic Erectile Dysfunction Rats. Stem Cells Int 2019:2168709. 10.1155/2019/2168709

Zhang HB, Chen FZ, He SH, Liang YB, Wang ZQ, Wang L, Chen ZR, Ding W, Zhao SC, and Wei AY. $2019 \mathrm{~b}$. In vivo tracking on longer retention of transplanted myocardin gene-modified adiposederived stem cells to improve erectile dysfunction in diabetic rats. Stem Cell Res Ther 10:208. 10.1186/s13287-019-1325-7

Zhang HB, Wang ZQ, Chen FZ, Ding W, Liu WB, Chen ZR, He SH, and Wei AY. 2017. Maintenance of the contractile phenotype in corpus cavernosum smooth muscle cells by Myocardin gene therapy ameliorates erectile dysfunction in bilateral cavernous nerve injury rats. Andrology 5:798-806. 10.1111/andr.12375

Zhang Z, and Zhang D. 2020. (-)-Epigallocatechin-3-Gallate Inhibits eNOS Uncoupling and Alleviates High Glucose-Induced Dysfunction and Apoptosis of Human Umbilical Vein Endothelial Cells by PI3K/AKT/eNOS Pathway. Diabetes Metab Syndr Obes 13:2495-2504. $10.2147 / \mathrm{dmso} . \mathrm{S} 260901$

Zhu GQ, Jeon SH, Bae WJ, Choi SW, Jeong HC, Kim KS, Kim SJ, Cho HJ, Ha US, Hong SH, Lee JY, Kwon EB, and Kim SW. 2018. Efficient Promotion of Autophagy and Angiogenesis Using Mesenchymal Stem Cell Therapy Enhanced by the Low-Energy Shock Waves in the Treatment of Erectile Dysfunction. Stem Cells Int 2018:1302672. 10.1155/2018/1302672 


\section{Figure 1}

Checking of RNA Quality, normalizing of GEO dataset, and screening of differentially expressed autophagy-related genes (ARGs).

(A) RNA degradation plot. (B) Boxplot of the relative logarithmic expression (RLE) reflected the consistency of parallel experiments. (C) Volcano plots showed the differentially expressed genes (DEGs) from GSE10804. Data points in red represent down-regulated, and green represent up-regulated genes. (D) Venn diagram was performed to screen the ARGs from the DEGs and autophagy-related genes. (E) Heatmap showed the 20 ARGs expression in ED and non-ED groups. 


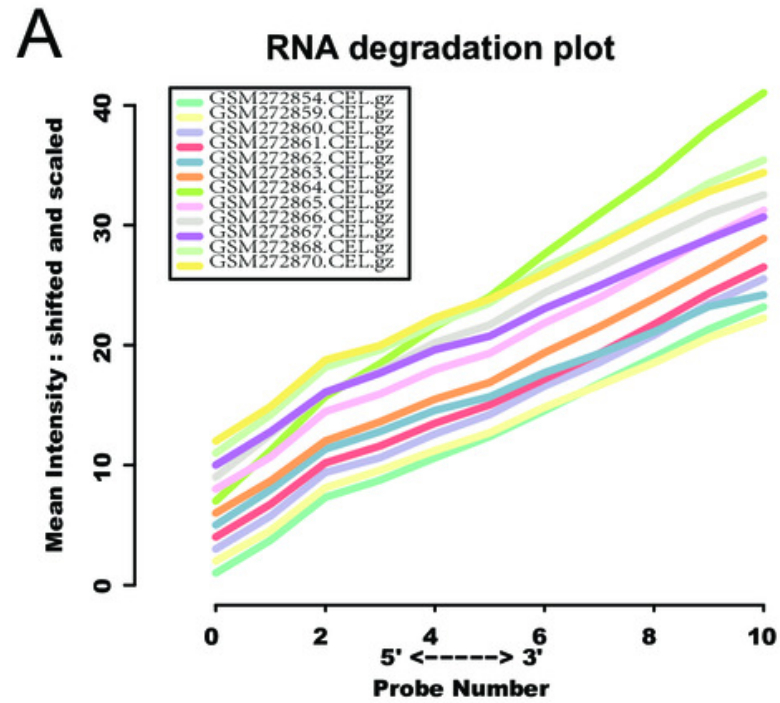

C ED group vs non_ED group

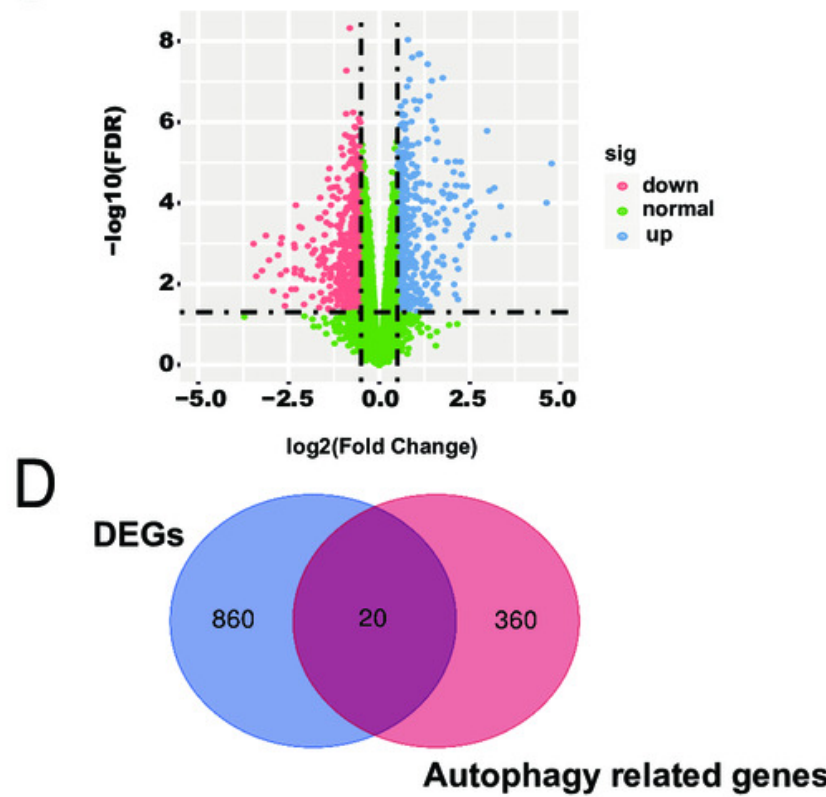

B Quality control chart of logarithmic expression of Data

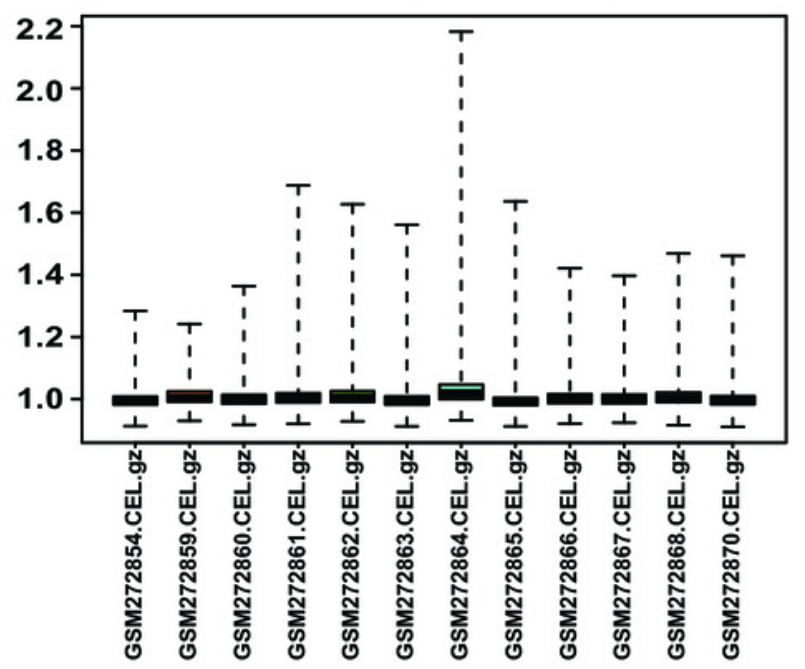

$E$

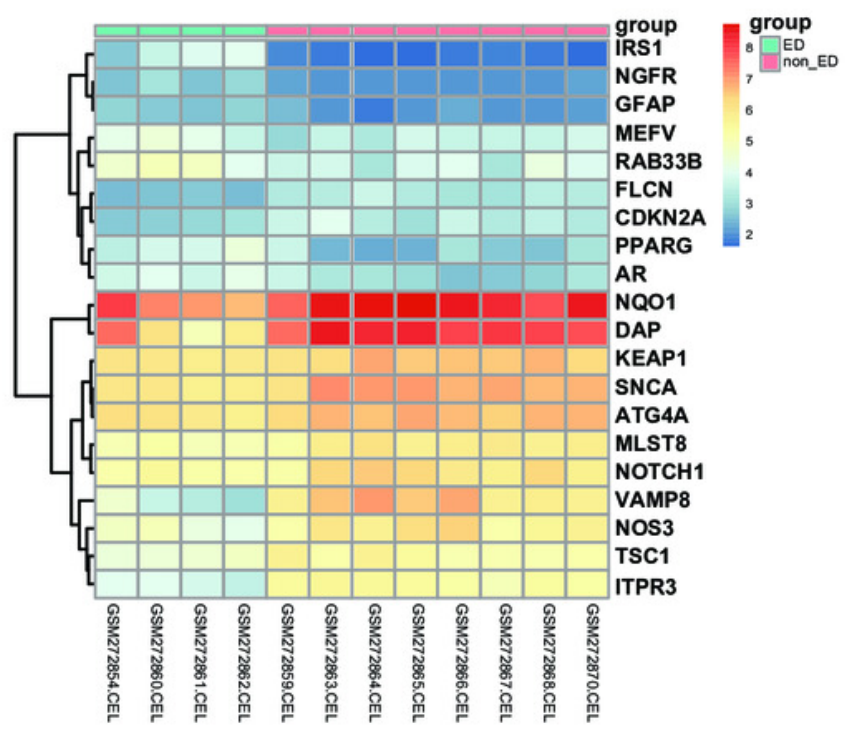




\section{Figure 2}

Gene Ontology (GO) enrichment analysis and KEGG pathway analysis.

(A) GO enrichment significance items of 20 ARGs were shown with bar plot in three functional groups: molecular function (MF), biological processes (BP), and cell composition (CC). The $x$-axis label represents the gene ratio, and the $y$-axis label represents $G O$ terms. (B) KEGG pathway analysis was performed with bar plot. (C) The centplot for the MF data in GO analysis was shown. (D) GO enrichment significance items of 7 up-regulated ARGs were shown with bar plot in two functional groups: MF and BP. The $\mathrm{x}$-axis label represents the gene ratio, and the $y$-axis label represents GO terms. (E) GO enrichment significance items of 13 down-regulated ARGs were shown with bar plot in three functional groups: MF, BP, and CC. The $x$-axis label represents the gene ratio, and the y-axis label represents GO terms. (F) GO enrichment significance items of 380 autophagy genes were shown with bar plot in three functional groups: MF, BP, and CC. The $x$-axis label represents the gene ratio, and the $y$-axis label represents $\mathrm{GO}$ terms. 


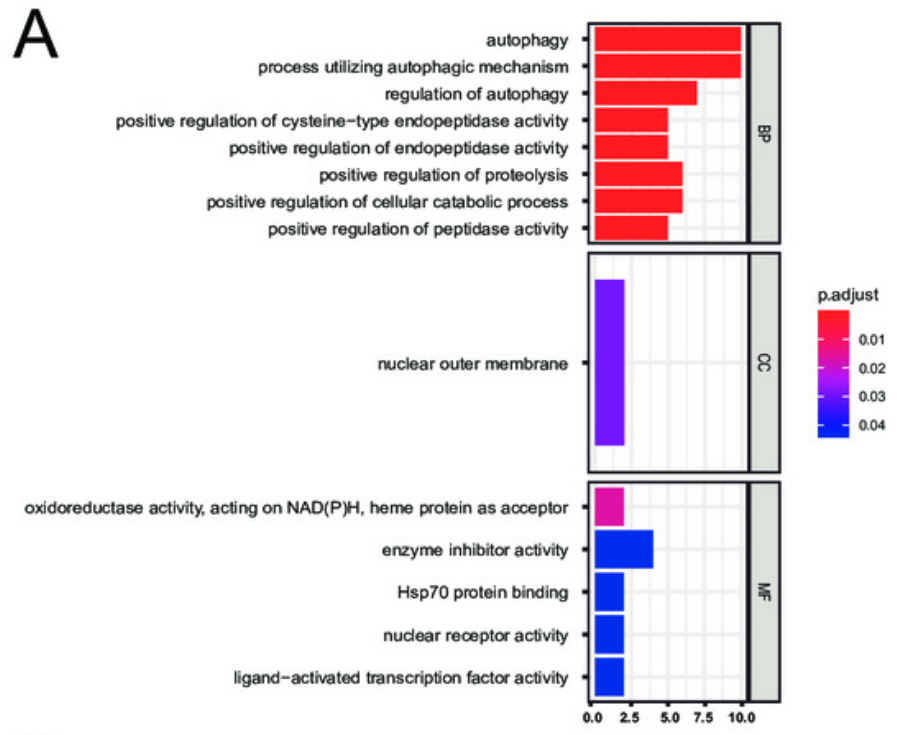

C
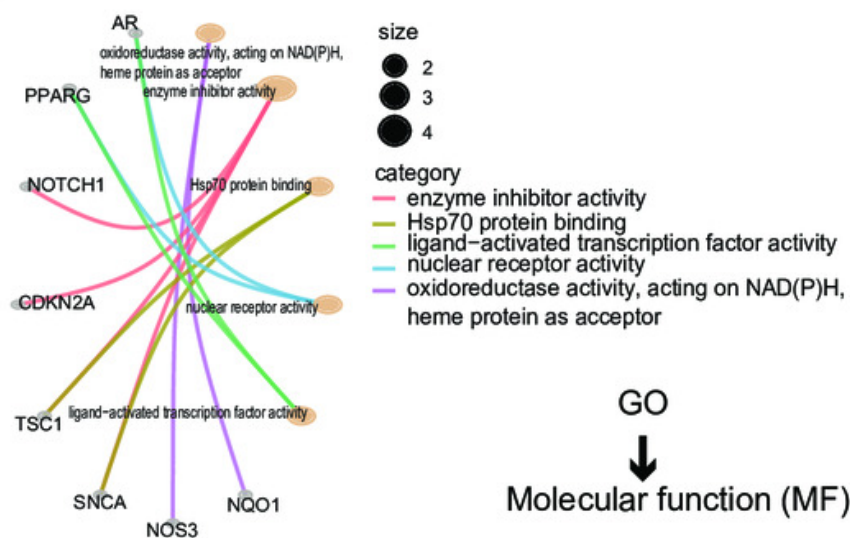

heme protein as acceptor

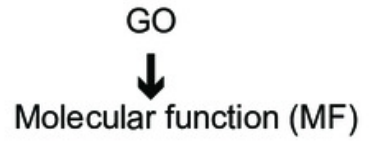

B

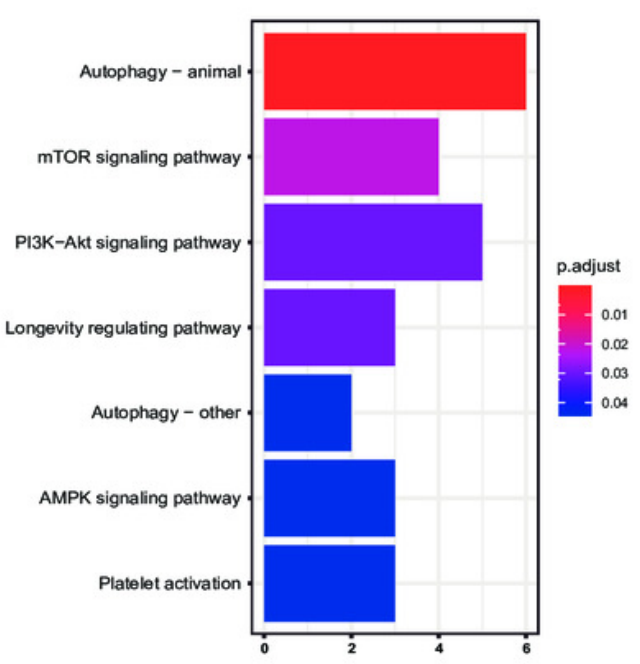

D

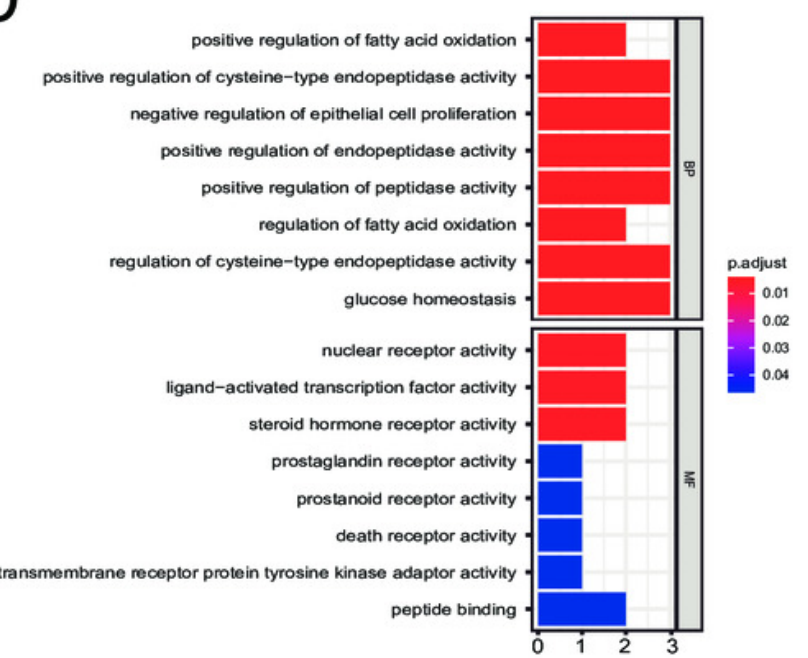

$F$

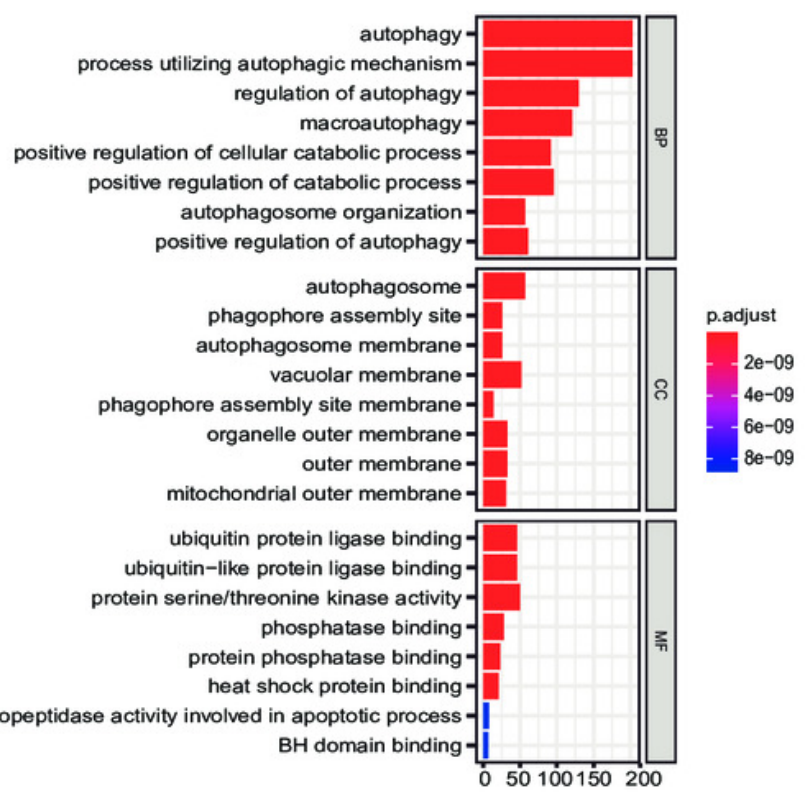




\section{Figure 3}

Gene interactions and enrichment of ARGs.

(A) The gene network downloaded from the STRING database indicated the interactions among the ARGs. (B) PPI network of ARGs by Cytoscape. Node color reflects the degree of connectivity (dark-green color represents a higher degree, and light-green color represents a lower degree)-networks of (C) target gene-miRNA and (D) target gene-TF. The red circle nodes are the genes, green hexagon nodes are the miRNAs, and green diamond nodes are the TFs. 
A

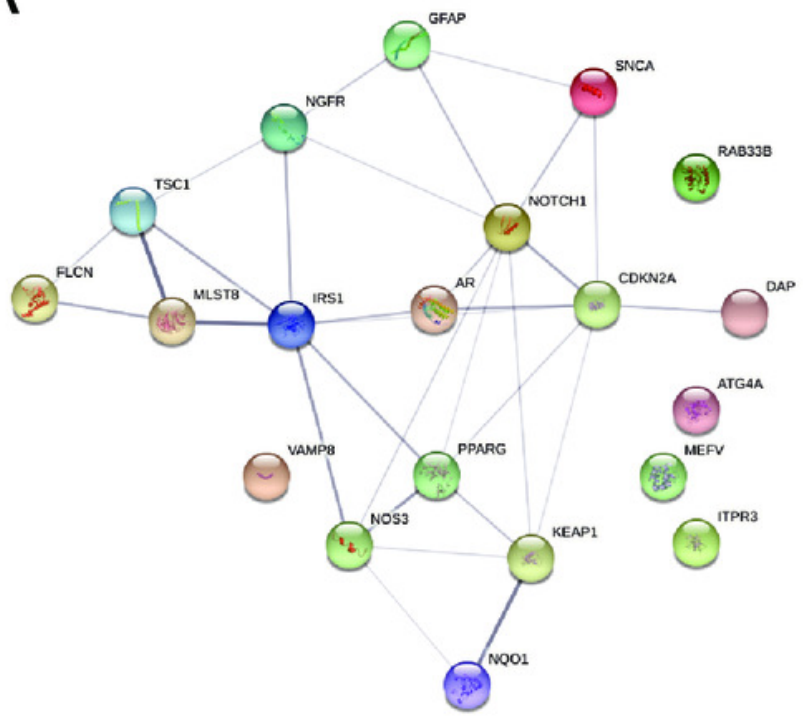

C

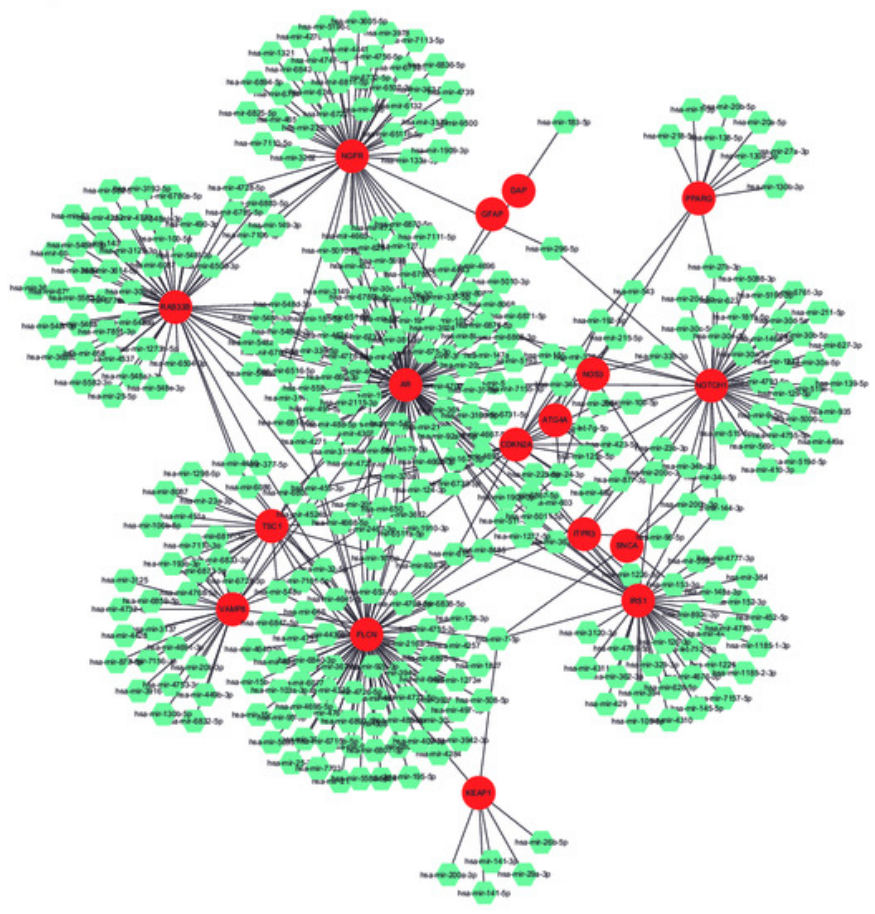

B

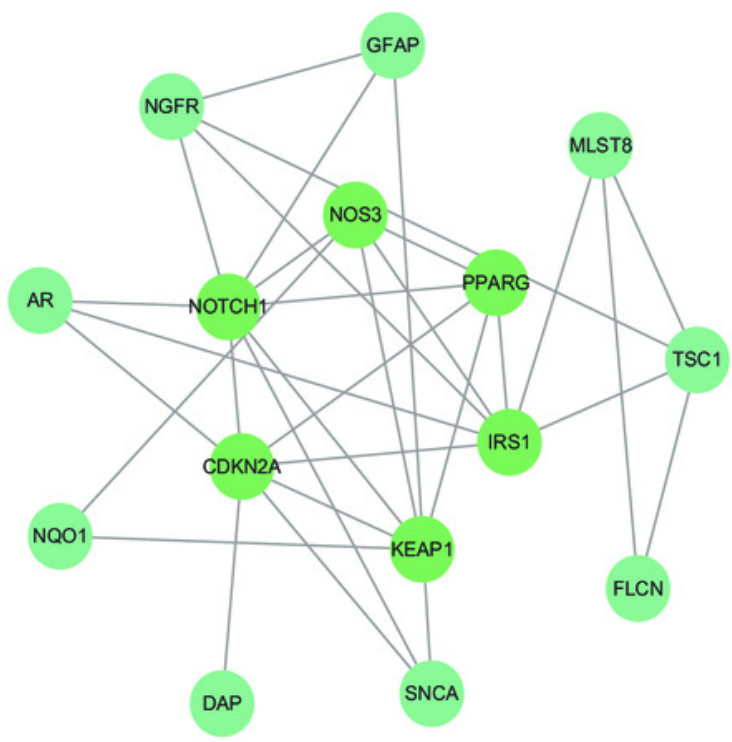

D

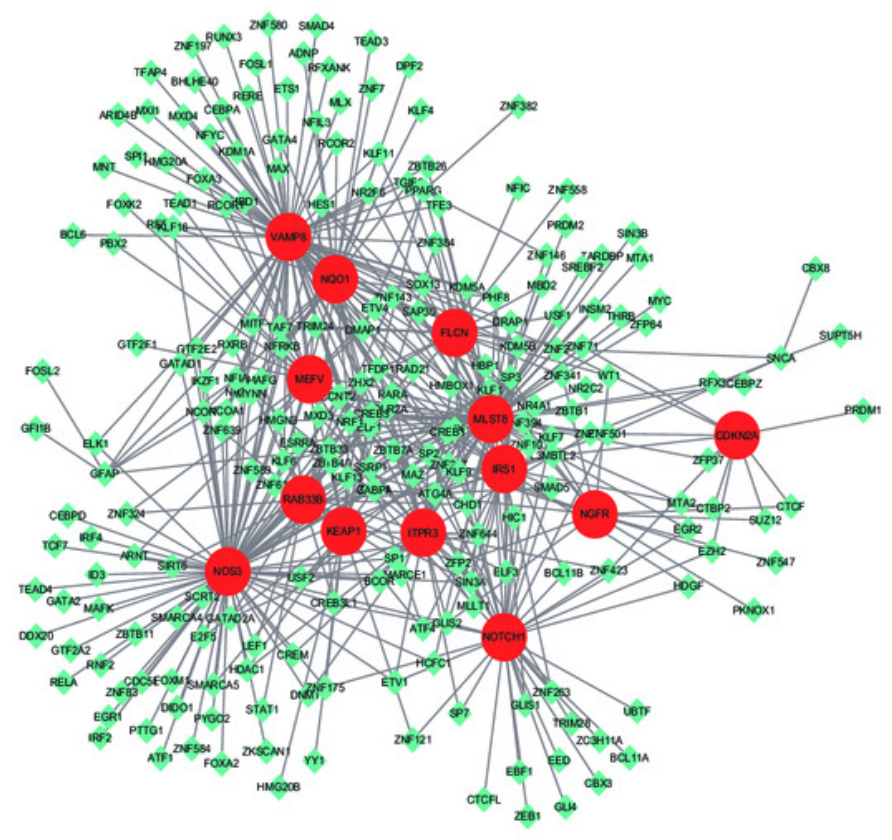




\section{Figure 4}

Establishment of DMED rat model.

(A) Bodyweight after 8 weeks' feeding. (b) blood glucose level in each group. Each bar represents mean \pm SEM. (B) Results of erectile function expressed as ICP and the ratio of ICP/MAP. Erectile function decreased significantly in the DMED group. Expressed as ICP/MAP ratio. (C) and (D) H\&E and Masson's trichrome staining showed significant differences in morphology and Smooth Muscle-to-collagen ratio among the different groups. $\mathrm{n}=3$ rats per group. $* p<0.05, * * p<0.01, * * * p<0.001$. 
A
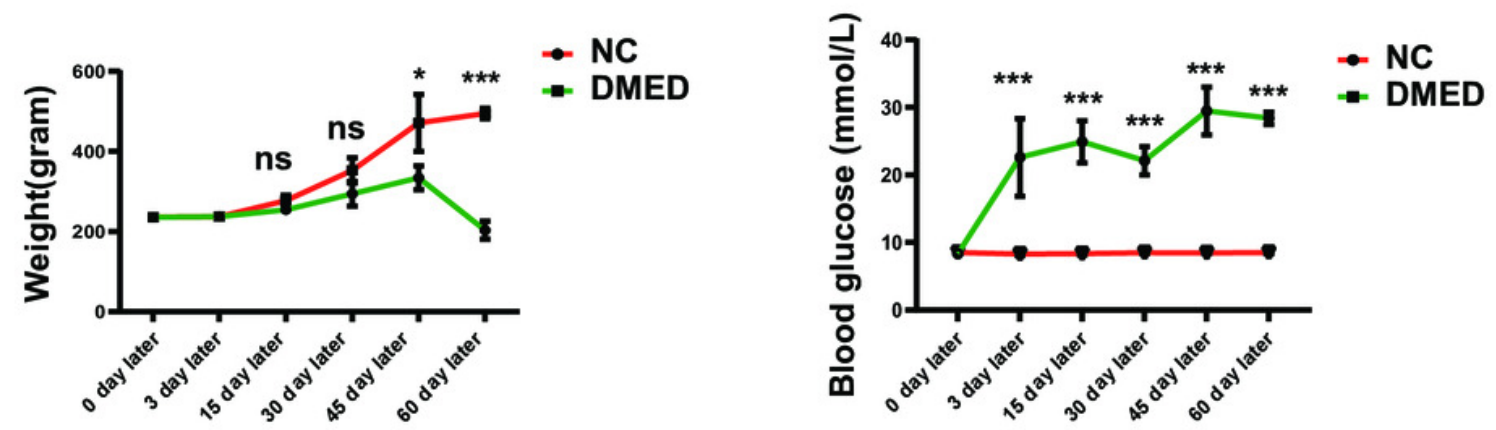

B
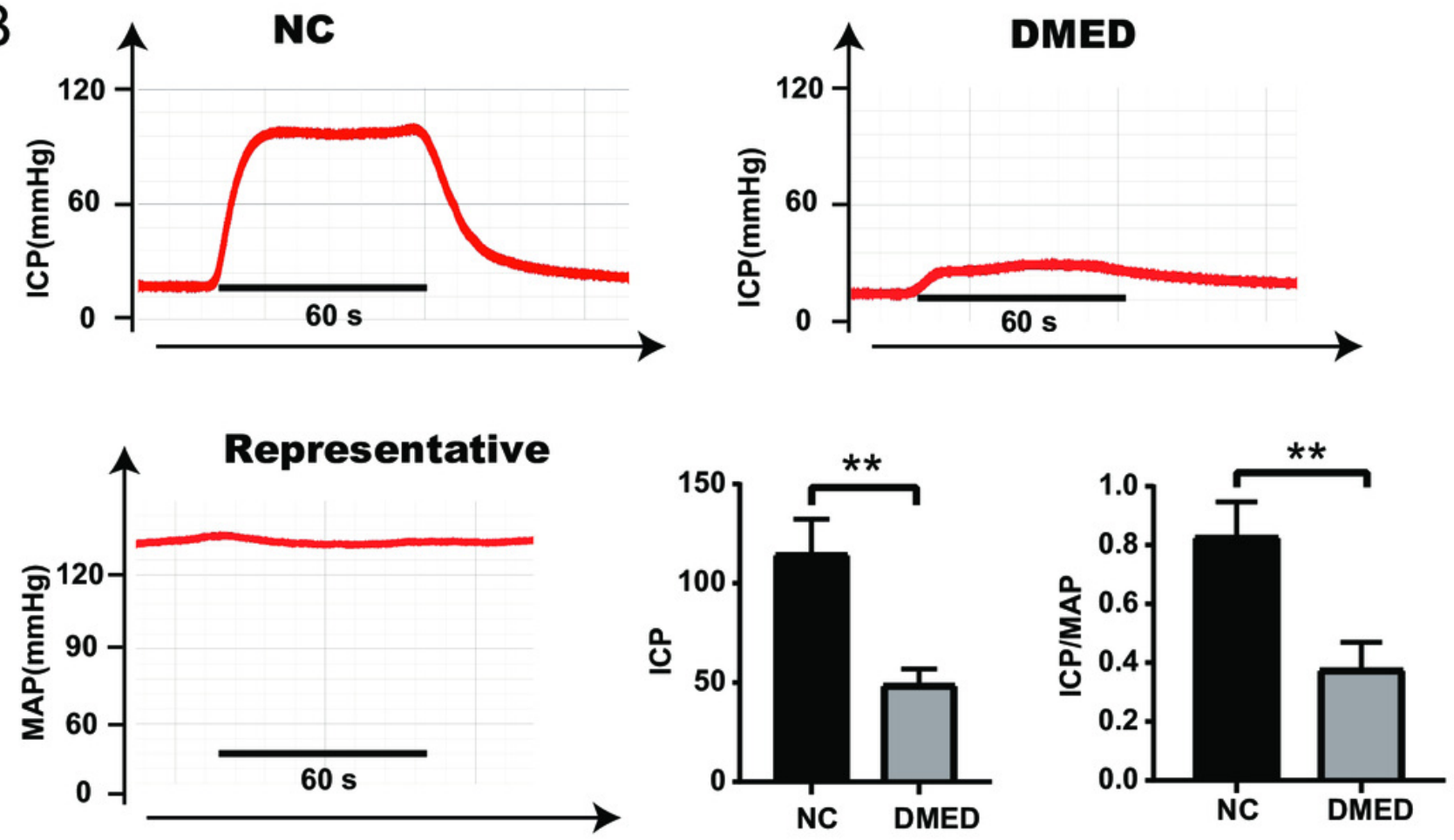

$\mathcal{C}$
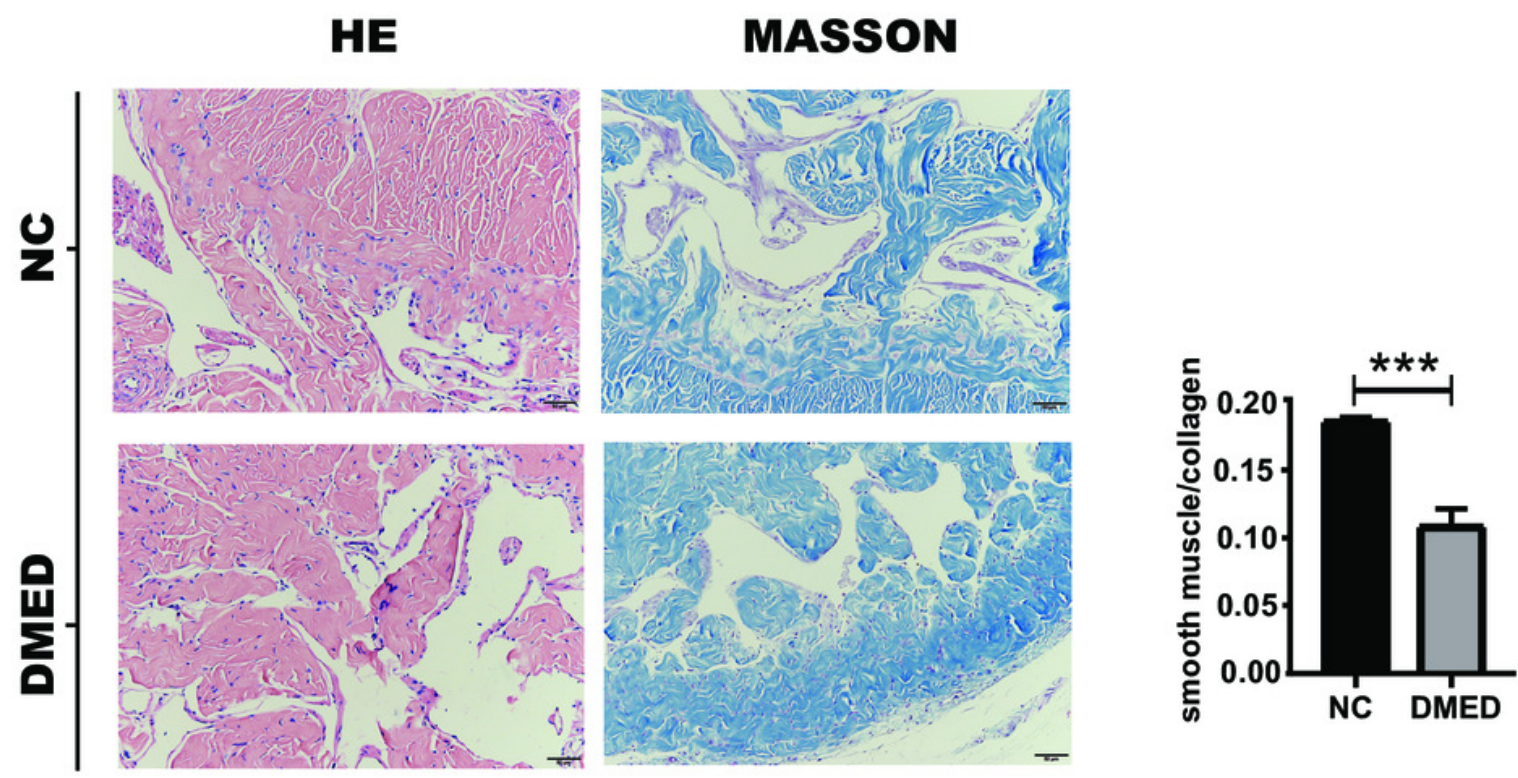
Figure 5

Autophagy increased, and endothelium was injured in the DMED rat model.

(A) Western blot showed that significantly increased LC3II, LC3I, LC3II/LC3I ratio, and reduced Beclin-1 expression were detected in DMED groups. (B) Immunofluorescence staining for CD31 and LC3 in vascular endothelial cells of corpus cavernosum in each group (Yellow arrow points to the sinus). Animals tested: $n=6 .{ }^{*} p<0.05$. 
A

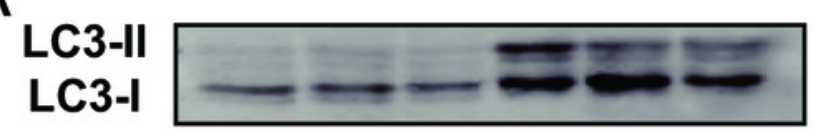

Beclin-1

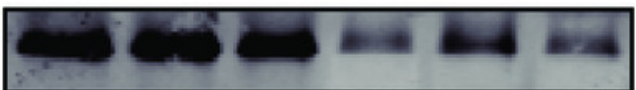

$\beta$-actin
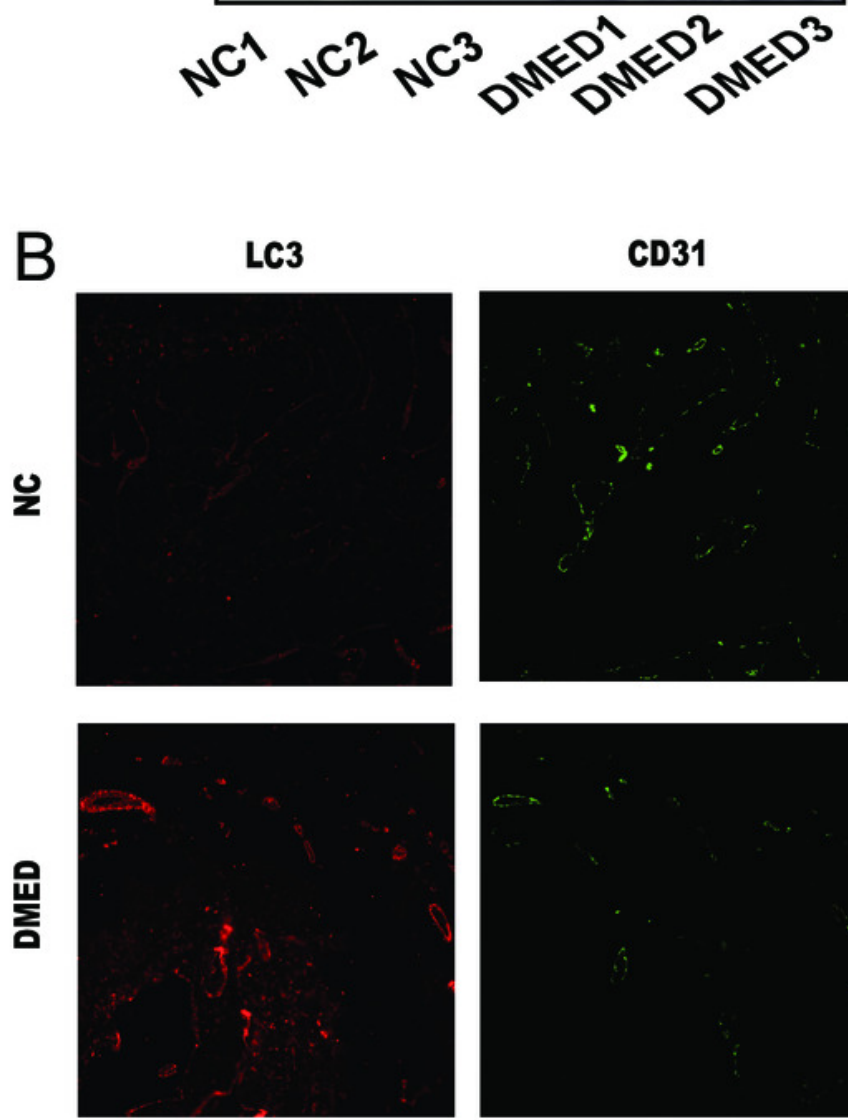
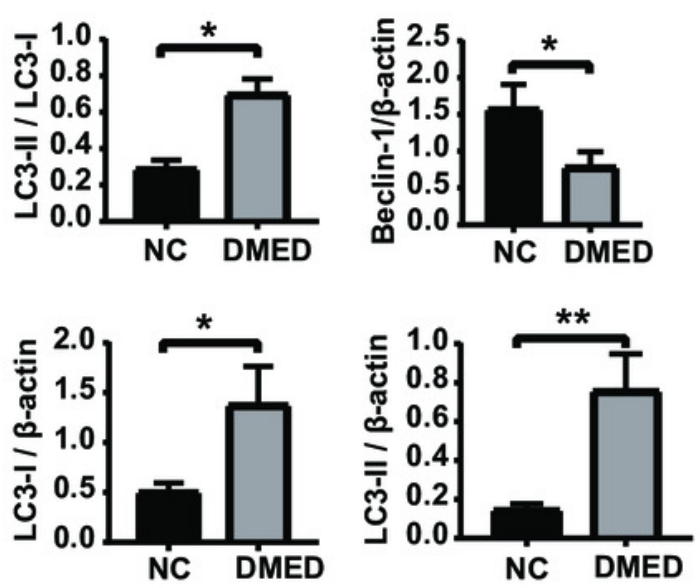

Merge
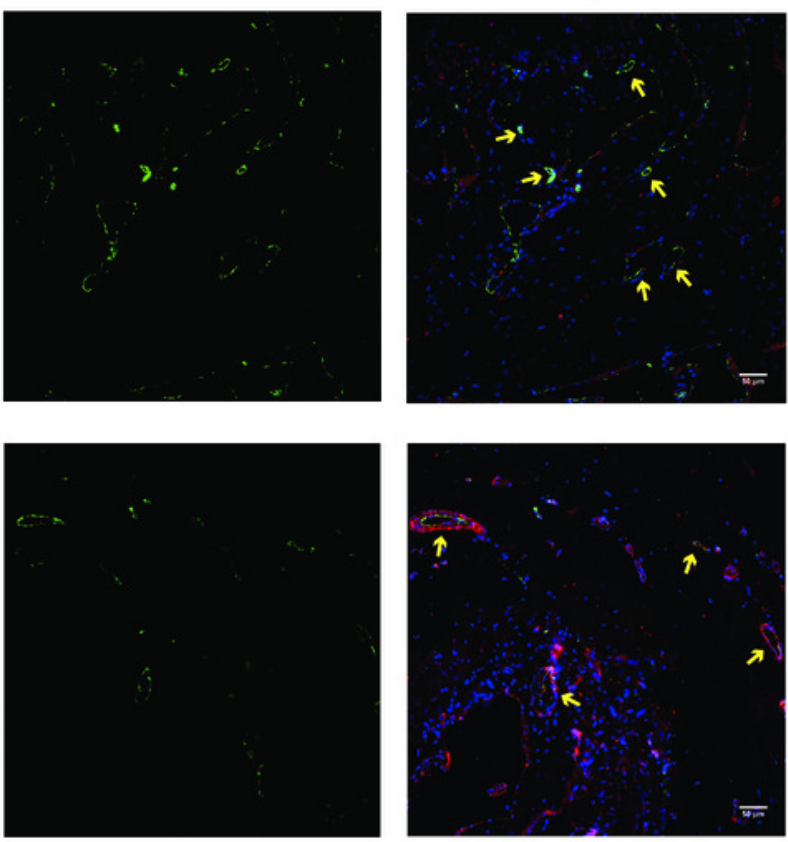
Figure 6

Identification of Hub ARGs with DMED model by qRT-PCR and WB.

(A) expression of NOTCH1, (B) expression of PPARG, (C) expression of NOS3, (D) expression of KEAP1, (E) expression of CDKN2A, (F) expression of IRS1, (G) protein level of CDKN2A, NOTCH1, and eNOS. ns: No significancy, ${ }^{*} p<0.05,{ }^{* *} p<0.01,{ }^{* * *} p<0.001$. 
A

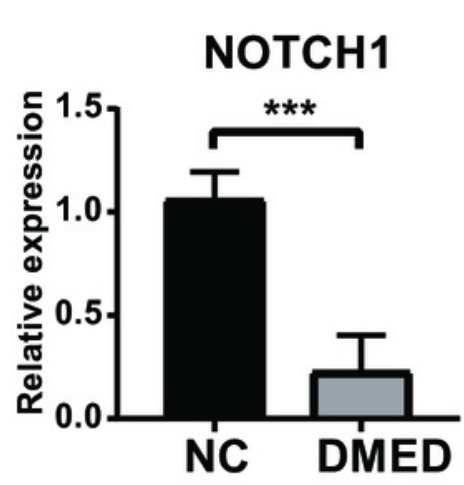

$\mathrm{D}$

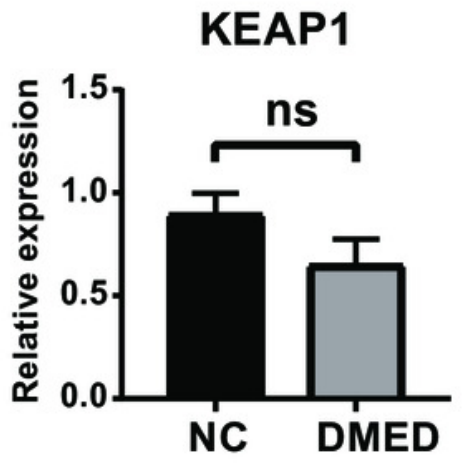

G

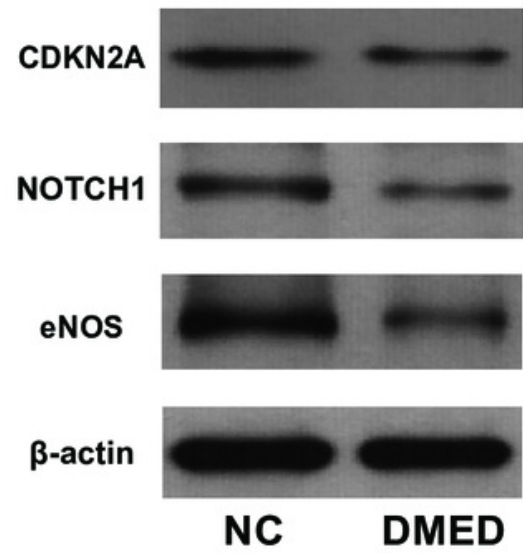

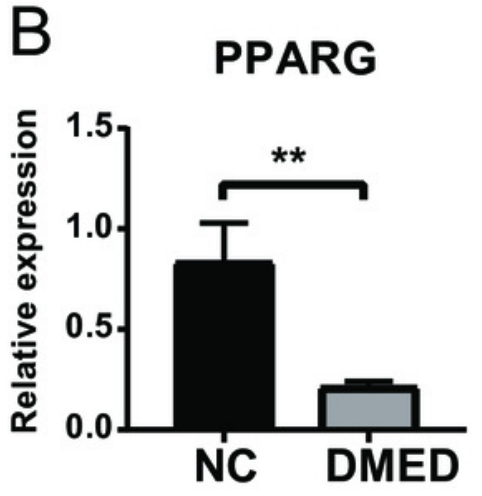

$E$

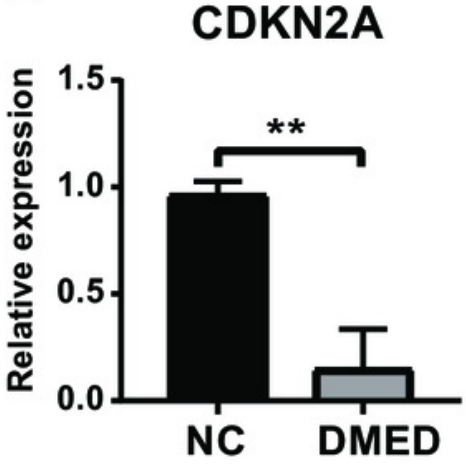

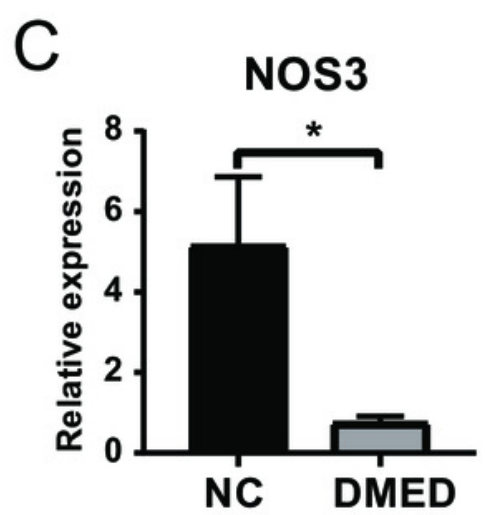

F

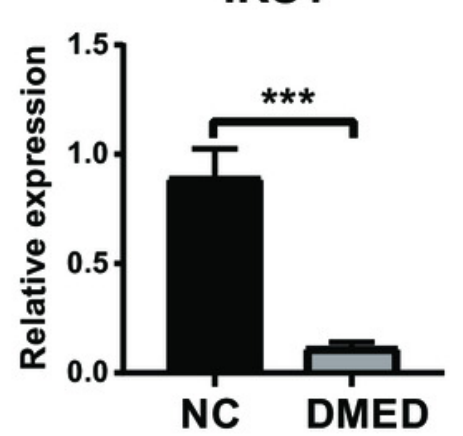

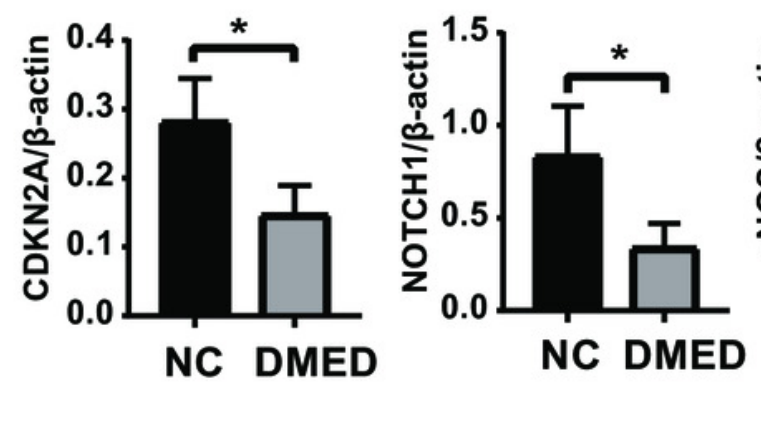

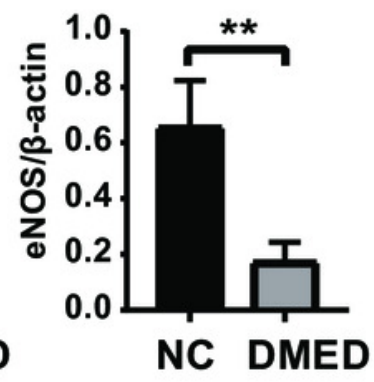


Table $\mathbf{1}$ (on next page)

Erectile dysfunction datasets (GSE10804) 
Table 1. Erectile dysfunction datasets (GSE10804)

\begin{tabular}{|c|c|c|c|c|}
\hline Simple & Origin & $\begin{array}{c}\text { Source } \\
\text { name }\end{array}$ & Cell type & $\begin{array}{c}\text { Used in } \\
\text { comparison }\end{array}$ \\
\hline GSM272854 & corpus cavernosum & HCCEC & HCCEC from donor with ED & ED \\
\hline GSM272859 & corpus cavernosum & HCCEC & $\begin{array}{l}\text { HCCEC from donor without } \\
\text { ED }\end{array}$ & Non_ED \\
\hline GSM272860 & corpus cavernosum & HCCEC & HCCEC from donor with ED & ED \\
\hline GSM272861 & corpus cavernosum & HCCEC & HCCEC from donor with ED & ED \\
\hline GSM272862 & corpus cavernosum & HCCEC & HCCEC from donor with ED & ED \\
\hline GSM272863 & Umbilical vein & HUVEC & $\begin{array}{l}\text { HUVEC from donor without } \\
\text { ED }\end{array}$ & Non_ED \\
\hline GSM272864 & Umbilical vein & HUVEC & $\begin{array}{l}\text { HUVEC from donor without } \\
\text { ED }\end{array}$ & Non_ED \\
\hline GSM272865 & Umbilical vein & HUVEC & $\begin{array}{l}\text { HUVEC from donor without } \\
\text { ED }\end{array}$ & Non_ED \\
\hline GSM272866 & Coronary artery & HCAEC & $\begin{array}{l}\text { HCAEC from donor without } \\
\text { ED }\end{array}$ & Non_ED \\
\hline GSM272867 & Coronary artery & HCAEC & $\begin{array}{l}\text { HCAEC from donor without } \\
\text { ED }\end{array}$ & Non_ED \\
\hline GSM272868 & Coronary artery & HCAEC & $\begin{array}{l}\text { HCAEC from donor without } \\
\text { ED }\end{array}$ & Non_ED \\
\hline GSM272870 & Coronary artery & HCAEC & $\begin{array}{l}\text { HCAEC from donor without } \\
\text { ED }\end{array}$ & Non_ED \\
\hline
\end{tabular}

2 ED: erectile dysfunction; non_ED: without erectile dysfunction 


\section{Table 2 (on next page)}

Significantly different expressed levels of ARGs inED andnon-ED tissues 
Table 2 Significantly different expressed levels of ARGs in ED and non-ED tissues

\begin{tabular}{ccccccc}
\hline Gene & $\operatorname{logFC}$ & AveExpr & $\mathrm{t}$ & P.Value & adj.P.Val & $\mathrm{B}$ \\
\hline MEFV & 0.583129 & 3.692823 & 3.142959 & 0.008025 & 0.046334 & -2.76814 \\
VAMP8 & -2.33546 & 5.195704 & -4.11645 & 0.001289 & 0.016228 & -0.97383 \\
RAB33B & 0.848874 & 4.013352 & 3.834705 & 0.002174 & 0.021723 & -1.48961 \\
IRS1 & 1.483492 & 2.269639 & 5.593557 & $9.72 \mathrm{E}-05$ & 0.003723 & 1.587609 \\
PPARG & 1.056873 & 3.134478 & 4.170111 & 0.001168 & 0.015245 & -0.8763 \\
NGFR & 0.670848 & 2.323239 & 7.183001 & $8.39 \mathrm{E}-06$ & 0.001237 & 3.993815 \\
SNCA & -0.85481 & 6.485949 & -5.42034 & 0.00013 & 0.004494 & 1.30249 \\
KEAP1 & -0.51936 & 6.318866 & -3.9344 & 0.001805 & 0.019673 & -1.30647 \\
ATG4A & -0.56943 & 6.423723 & -5.12685 & 0.000213 & 0.005868 & 0.809451 \\
FLCN & -0.75199 & 3.080793 & -7.52722 & $5.16 \mathrm{E}-06$ & 0.001042 & 4.465874 \\
TSC1 & -0.67593 & 4.957076 & -4.42259 & 0.000737 & 0.011674 & -0.42107 \\
NQO1 & -1.12484 & 7.957864 & -4.21921 & 0.001067 & 0.014561 & -0.78729 \\
MLST8 & -0.7294 & 5.552902 & -5.46794 & 0.00012 & 0.004307 & 1.381291 \\
NOS3 & -1.13072 & 5.27702 & -3.47571 & 0.004269 & 0.032331 & -2.15277 \\
GFAP & 0.598847 & 2.32642 & 5.327376 & 0.000152 & 0.004764 & 1.147666 \\
DAP & -0.53841 & 7.869929 & -3.34197 & 0.0055 & 0.037284 & -2.40038 \\
CDKN2A & -0.54667 & 3.220725 & -3.4103 & 0.004831 & 0.034619 & -2.27388 \\
AR & 0.879528 & 3.323439 & 4.801902 & 0.000375 & 0.008083 & 0.249554 \\
NOTCH1 & -0.78118 & 5.779909 & -3.66608 & 0.002981 & 0.026172 & -1.80058 \\
ITPR3 & -1.35676 & 4.747004 & -6.09948 & $4.29 \mathrm{E}-05$ & 0.002502 & 2.394674 \\
\hline
\end{tabular}

2 LogFC: $\log$ fold change, adj.P.Val: adjust P value 